\title{
Optimization of blade profiles for the Wells turbine
}

\author{
Tim Gratton ${ }^{\mathrm{a}}$, Tiziano Ghisu ${ }^{\mathrm{b}, *}$, Geoff Parks $^{\mathrm{a}}$, Francesco Cambuli ${ }^{\mathrm{b}}$, Pierpaolo \\ Puddu ${ }^{\mathrm{b}}$ \\ ${ }^{a}$ Department of Engineering, University of Cambridge, Trumpington Street, Cambridge, \\ CB2 1PZ, United Kingdom \\ ${ }^{b}$ Department of Mechanical Engineering, Chemistry \& Materials, University of Cagliari, \\ Cagliari 09123, Italy
}

\begin{abstract}
A Wells turbine, when coupled with an oscillating water column, allows the generation of power from the energy in waves on the surface of the ocean. In the present work, a tabu search is used to control the process of optimising the blade profile in the Wells turbine for greater performance, by maximising the torque coefficient. A free form deformation method is used as an efficient means of manipulating the blade profile and computational fluid dynamics in OpenFOAM are used to assess each profile in both two and three dimensions. Investigations into both the flow coefficient at which the optimisation is performed and the number of control variables in the free form deformation tool are performed before optimisations are done on a two-dimensional blade at the hub and tip solidities. This results in increases to the torque coefficient of $34 \%$ and $32 \%$ at the tip and hub solidities, respectively. These results are then applied to the three-dimensional turbine, giving a $14 \%$ increase in the torque coefficient. The results are assessed and an improved method of optimising the blade in two dimensions is proposed.
\end{abstract}

Keywords: Wells turbine, Optimization, OpenFOAM, Tabu search, Free form deformation

\footnotetext{
${ }^{*}$ Corresponding author

Email address: t.ghisu@unica.it (Tiziano Ghisu)
} 


\section{Introduction}

As the world moves into an age where it can no longer be reliant on fossil fuels to meet our energy needs, renewable sources of electrical power are being explored more thoroughly than they have been in the past. While solar and wind energy is available around $20-30 \%$ of the time, energy from waves on the surface of the ocean is available up to $90 \%$ of the time in a suitable location [1]. One method of harvesting this energy is through the use of oscillating water column (OWC) systems equipped with a Wells turbine, proposed by Prof. Alan Arthur Wells [2].

A reciprocating airflow through a duct is produced when waves reach an OWC system built on the shore [3, 4] or floating in water [5]. A turbine installed in the duct creates a torque that is used to drive a generator which produces electrical power. Due to the nature of the task, the turbine must be able to generate power from airflow in both directions (inflow and outflow) and, as a result of this, it usually has a symmetric layout. The rotation of the blade combined with the axial flow effectively create a wing at incidence, and the associated lift and drag forces can be resolved in the axial $\left(F_{A}\right)$ and tangential $\left(F_{T}\right)$ directions. The Wells turbine therefore shares many of the features of a symmetric wing: the low lift at low angles of attack requires the axial flow to be sufficiently large for the lift component of the tangential force to overcome the drag in order to produce power. There is also an upper limit, above which stall reduces the tangential force. These two features of the Wells turbine limit the values of the flow coefficient $(\phi)$, defined as the ratio of axial velocity to blade speed, where the device is useful to those immediately preceding stall.

Increasing the performance of the Wells turbine would provide greater power output to the electrical grid for a device of the same size. There are two key measures of the performance of the Wells turbine: non-dimensional torque $\left(C_{T}\right)$ and efficiency $(\eta)$ which are defined in equations 1 13 . The non-dimensional torque, or torque coefficient, is a measure of the useful work produced by the turbine blades. An increase in the torque $(T)$ supplied to the generator will 
allow more power to be produced. The efficiency takes the torque coefficient and divides it by the non-dimensional axial force on the blade, a measure of the pressure drop across it, and the flow coefficient. If the efficiency is increased, it means that, for the same pressure drop, there is a greater torque driving the generator. This parameter is important, as if in the process of increasing the torque coefficient the efficiency is lowered, a greater pressure difference will be required across the blade. Due to effects in the OWC, the peak axial flow velocity will be reduced, decreasing the peak flow coefficient and subsequently the peak torque. This would negate some of the improvements that have been made [6].

$$
\begin{aligned}
C_{T} & =\frac{T}{\frac{1}{2} \rho \Omega^{2} r_{\mathrm{tip}}^{5}} \\
C_{A} & =\frac{\Delta p}{\frac{1}{2} \rho \Omega^{2} r_{\mathrm{tip}}^{2}} \\
\eta & =\frac{C_{T}}{C_{A} \phi} \frac{1}{\pi\left(1-\frac{r_{h}^{2}}{r_{t}^{2}}\right)}
\end{aligned}
$$

There are many parameters that can affect the performance of the Wells turbine. A summary of previous studies on the effects of blade sweep, solidity and profile is presented below.

Three-dimensional (3D) simulations in ANSYS Fluent [7] have been used to consider the effect of sweep on a Wells turbine blade with both NACA0020 and CA9 profiles by Kim et al. [8]. A blade sweep ratio of 0.35 was found to improve the mean efficiency and stall point, and the NACA0020 profile was found to perform better than the CA9 profile. A formal optimization on the blade sweep parameter at the mid and tip sections has been completed by Halder et al. [9]. Simulations of a monoplane turbine with a NACA0015 profile were done in $A N S Y S C F X$ [10] and used to create surrogate models for the efficiency and torque coefficient. These models were then used with a genetic algorithm to identify a Pareto-optimal front for the problem. Two designs were selected for evaluation: the first increased the torque coefficient by $28 \%$ and the operating 
range by $18 \%$ at the expense of a $14 \%$ decrease in efficiency, while the second increased the efficiency by $6 \%$ but decreased the peak torque coefficient by $36 \%$ and decreased the operating range by $22 \%$. These results illustrate the trade-off that is often found in real-world multi-objective optimization problems.

A computational investigation using the NEWT solver [11] on three different solidities of Wells turbine has been presented by Watterson and Raghunathan [12]. This work found that increasing the solidity of the turbine increased the operating range. A new, variable chord length, Wells turbine design is suggested by Soltanmohamadi and Lakzian [13]. A blade profile varying from NACA0020 at the hub to NACA0012 at the tip is used for aerodynamic and structural reasons. A $26 \%$ reduction in entropy generation was achieved. Solidity was further considered by Shaaban [14] by combining a genetic algorithm with computational fluid dynamics in ANSYS Fluent. Pareto solutions were found that could either increase the efficiency by up to $5 \%$ with a $3 \%$ decrease in torque coefficient or increase the torque coefficient by $11 \%$ while also increasing the efficiency by $2 \%$.

Studies into the effects of the blade profile on the turbine performance have been also carried out. Among standard symmetric profiles, the NACA0020 blade appears the most suitable for low Reynolds number turbines [15] while the NACA0015 profile displayed better performance at larger Reynolds numbers; however, due to the nature of the experimental approach taken, these studies were only able to consider four blades from standard families. To the authors' knowledge, the only work that studies the use of generic blade profiles (i.e. not from standard families) in a Wells turbine is the one from Mohamed et al. [16], who combine a multi-objective genetic algorithm with a parameterization of the blade profile using a spline fit of a series of 12 control points. The evaluations were completed using two-dimensional (2D) simulations in ANSYS Fluent. This led to an increase in torque coefficient of $12 \%$ and a $1 \%$ increase in efficiency for the optimized blade when compared to the datum NACA0021 profile. No verification of the applicability of these results to the 3D turbine is presented. A similar optimization algorithm has been used by Shaaban [17], but applied 
to the biplane turbine, using a 3D evaluation in ANSYS Fluent. Due to the significantly larger time require by $3 \mathrm{D}$ simulation, the description of the blade profile has been simplified significantly, by dividing a NACA0015 profile at the point of maximum thickness, and allowing only a scaling the two sections. This led to a $9 \%$ improvement in the peak torque coefficient.

This work presents an optimization of the blade profiles for a Wells turbine, using a free form deformation tool to modify the blade shape and a tabu search optimization algorithm to explore the design space. The objective is to maximise the torque coefficient, as evaluated by computational fluid dynamics (CFD). Initially, an investigation is conducted to find the optimal number of control variables in the free form deformation (FFD) tool. Then, the profiles used for the hub and tip sections are optimized in 2D. A 3D turbine has been generated by applying a linear variation of the hub and tip profiles and then evaluated to verify the actual gain in performance. A choice has been made to keep the blade solidity fixed at the datum value, in order to avoid a significant variation of the pressure drop vs flow coefficient curve (i.e. the turbine damping), which affects the water level displacement in the OWC and therefore the turbine working conditions [18, 6, 19, 20, 21, 22].

The rest of the paper is organized as follows. Section 2 presents the optimization system (i.e. optimizer, parameterization approach, and evaluation system), Section 3 presents the results from the two-dimensional optimization of the blade profiles, and Section 4 verifies the proposed modifications in three dimensions. Finally, Section 5 presents some conclusions and suggestions for future work.

\section{Optimization system}

The optimization system involves three main components. A tabu search routine, described in Section 2.1, is used to explore the design space, represented by the control point locations that are used in the FFD tool presented in Section 2.2. These control points are used to modify the blade shape and, accordingly, the simulation domain. The performance of the new profile is then 
assessed using CFD to evaluate the objective function. This component is outlined in Section 2.3. A flow chart for this system is shown in Figure 1 .

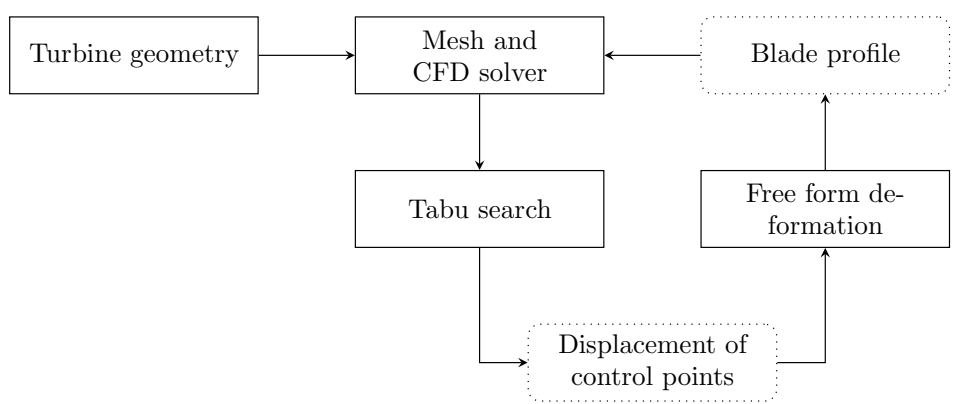

Figure 1: Interaction of the system components

\subsection{Tabu Search}

The tabu search (TS) algorithm [23] is a metaheuristic optimization method with a local search at its core, but with routines to escape local minima and explore further regions of the search space. While this method cannot guarantee that the global minimum will be found, the progressive nature of the search should ensure that some improvement is made, making it suitable for the task of optimising the blade profile of the Wells turbine. The algorithm is well suited to parallelization, by evaluating the objective function of each candidate nontabu move simultaneously across multiple computing nodes. This has a direct influence on the choice of CFD software that is best suited to the task. In previous research, the commercial code ANSYS Fluent has been used to great effect [24, 8]. However, licences must be purchased for each individual node that is to be used simultaneously and this would severely limit the extent to which the search could be carried out in parallel. Open source codes do not have this limitation, making them a better choice for this work. Of all the open source codes, OpenFOAM [25, 26] is the most well documented for a large number of applications and was chosen for this work.

The TS implementation by Jaeggi et al. [27, 28] allows the simultaneous optimization of multiple objective functions. Possible choices for the objective function(s) are the torque coefficient and the efficiency of the Wells turbine, as 
discussed in Section 1. Previous research attempts to maximise both have shown there to be little change in efficiency for large increases in torque coefficient [16], so the optimization problem has been set up as single-objective, aiming to maximise the torque coefficient at a specific flow coefficient. The impacts of this choice will be discussed later in the paper.

\subsection{Free form deformation}

The ability to change the geometry is at the heart of every design optimization problem. The choice of the parameterization scheme determines both the range of possible solutions and the intricacy of exploring the design space [29]. Given the same evaluation tools, the parameterization approach is what defines the optimization problem formulation and the design space topology [30].

A comprehensive survey of the different parameterization approaches available for shape optimization is given in 31]. These include: the use of typical engineering parameters, discrete approaches, basis vectors, domain element methods, polynomials and splines - both Bezier curves and non-uniform rational Bezier splines (NURBS), partial differential equations, CAD-based methods, and FFD. FFD is a subset of the soft object animation algorithms used in computer graphics for deforming surfaces and solid models [32]. Mathematically, it is defined in terms of a tensor product trivariate Bernstein polynomial:

$$
\begin{aligned}
\mathbf{X}_{F F D}= & \left(\sum_{i=0}^{l}\left(\begin{array}{l}
l \\
i
\end{array}\right)(1-s)^{l-i} s^{i}\right)\left(\sum_{j=0}^{m}\left(\begin{array}{c}
m \\
j
\end{array}\right)(1-t)^{m-j} t^{j}\right) \\
& \left(\sum_{k=0}^{n}\left(\begin{array}{l}
n \\
k
\end{array}\right)(1-u)^{n-k} u^{k}\right) \mathbf{P}_{i j k}
\end{aligned}
$$

where $\mathbf{X}_{F F D}$ is the deformed position of an arbitrary point with initial coordinates $(s, t, u)$. This approach assumes a lattice of $l \times m \times n$ initially equally spaced control points. The deformed positions of these nodes are defined by the vectors $\mathbf{P}_{i j k}$ [33].

FFD is capable of generating radically new shapes, but its generality can also lead to large design spaces in applications where specialized, highly efficient 
parameterization schemes can reduce the number of design variables considerably [34].

A 2D FFD tool has been written for the application of modifying aerofoil geometries. The first and last chord-wise points do not move in order to maintain the same solidity of the datum profile.There is also a horizontal mirror to the lattice deformation, in order to maintain the aerofoil symmetry. This means that a FFD using $N$ control points requires $N-4$ control variables, defining the change in the $x$ and $y$ coordinates of the central control points. An example of using this tool with eight control points and four control variables is shown in Figure 2, The moving forwards and widening of the lattice near the leading edge increased the thickness at the point of maximum thickness and moved its location forwards. The moving forwards and narrowing of the lattice nearer the trailing edge reduced the aerofoil thickness in this region.

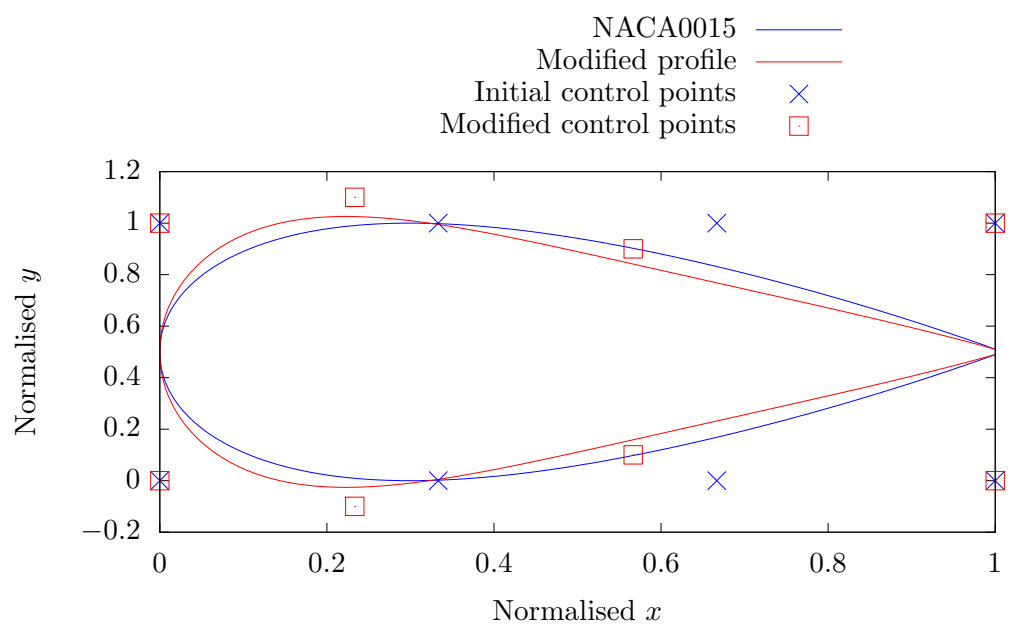

Figure 2: Example: free form deformation of the NACA0015 aerofoil

\subsection{Computational fluid dynamics}

\subsubsection{Mesh}

Before the CFD evaluation can be used, the domain must be discretised in a process known as mesh generation. The blockMesh [25] library of OpenFOAM 
was used for all meshes. A script was written to generate the dictionary file that controls the process. This used the datum turbine dimensions, shown in Table 1, and took in the blade profile created by the FFD tool.

\begin{tabular}{lc}
\hline Parameter & Value \\
\hline \hline Rotor tip diameter & $250 \mathrm{~mm}$ \\
Rotor hub diameter & $190 \mathrm{~mm}$ \\
Tip clearance & $1 \mathrm{~mm}$ \\
Chord length & $36 \mathrm{~mm}$ \\
Number of blades & 14 \\
Solidity at tip radius & 0.642 \\
Solidity at hub radius & 0.844 \\
Sweep ratio & 0.417 \\
Rotational speed & $4200 \mathrm{rpm}$ \\
\hline
\end{tabular}

Table 1: Wells turbine geometry corresponding to the experimental data of Puddu et al. 35.

In 3D, a single blade passage was meshed, in order to minimise the computational cost, as shown in Figure 3. A periodic boundary condition was set on the radial boundaries. A C-mesh was used around the blade, in order to accurately capture the boundary layer flow, while an H-mesh was used in the rest of the domain. The blockMesh library has limited support for such a mesh, which would naturally be represented in cylindrical coordinates, as the definitions are in Cartesian coordinates. A system was devised where the mesh was generated with the blockMesh $x, y, z$ Cartesian coordinates representing the $r, \theta, z$ cylindrical polar coordinates of the desired mesh. This gave the correct mesh connectivity between points, and the mesh was finalised by transforming the point locations with a secondary script. The domain used in the simulation extended to 8 blade chords both upstream and downstream of the blade.

In $2 \mathrm{D}$, a similar approach was used, except the mesh was one cell deep in the radial direction, with empty boundary conditions employed on the front and back faces and periodic conditions on either sides of the blade to allow the simulation of only one blade passage, while reproducing the effect of blade interaction. The moving reference frame was not used; instead, a tangential velocity component equal to the blade speed was used at the inlet. 


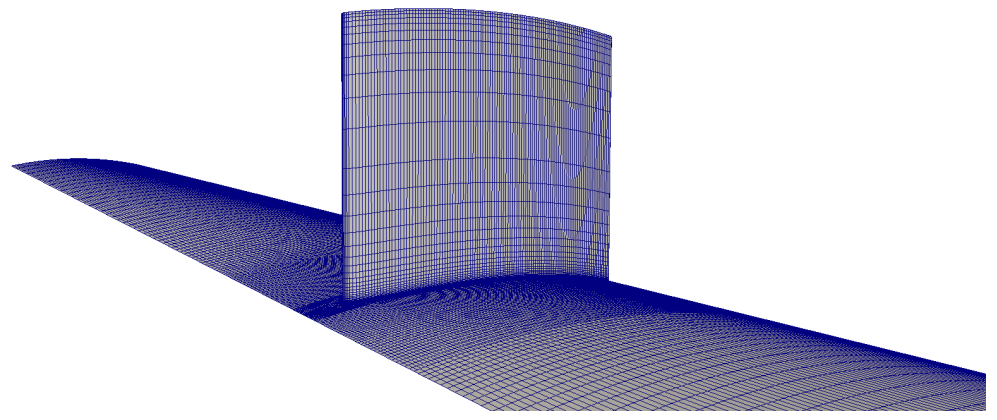

Figure 3: The blade and hub wall of the 3D mesh

A summary of the number of cells used in each direction can be found in Table 2. This gave a total of approximately 35,000 cells in 2D and 1,000,000 cells in 3D. A coarser and a finer mesh were generated, with a $30 \%$ decrease and increase in the cell count. The change in the torque coefficient with flow coefficient in two and three dimensions is shown in Figure 4 and given that the difference in torque coefficient was never greater than $3 \%$ for flow coefficients where the flow was attached, the medium mesh was chosen for both cases. The number of cells is similar to the ones used in several other studies [36, 37, 8].

\begin{tabular}{lc}
\hline Region & Number of cells \\
\hline \hline Around blade surface & 235 \\
Normal to blade & 55 \\
Between blades & 50 \\
Blade radial direction & 35 \\
Tip gap & 10 \\
\hline
\end{tabular}

Table 2: Summary of the number of cells in each direction

\subsubsection{Solver and numerical schemes}

The simpleFoam [25] solver of OpenFOAM was used to iterate the steady, incompressible Navier-Stokes equations with turbulence modelling. A steadystate solver was chosen as the computational cost is much lower than would be the case for an unsteady solver. This will be critical in allowing the TS to make 
2D tip sol. (NACA0015) [coarse mesh] - -

$2 \mathrm{D}$ tip sol. (NACA0015) [medium mesh] -

$2 \mathrm{D}$ tip sol. (NACA0015) [fine mesh] - -

3D (NACA0015) [coarse mesh] -.-

3D (NACA0015) [medium mesh]

3D (NACA0015) [fine mesh] - -

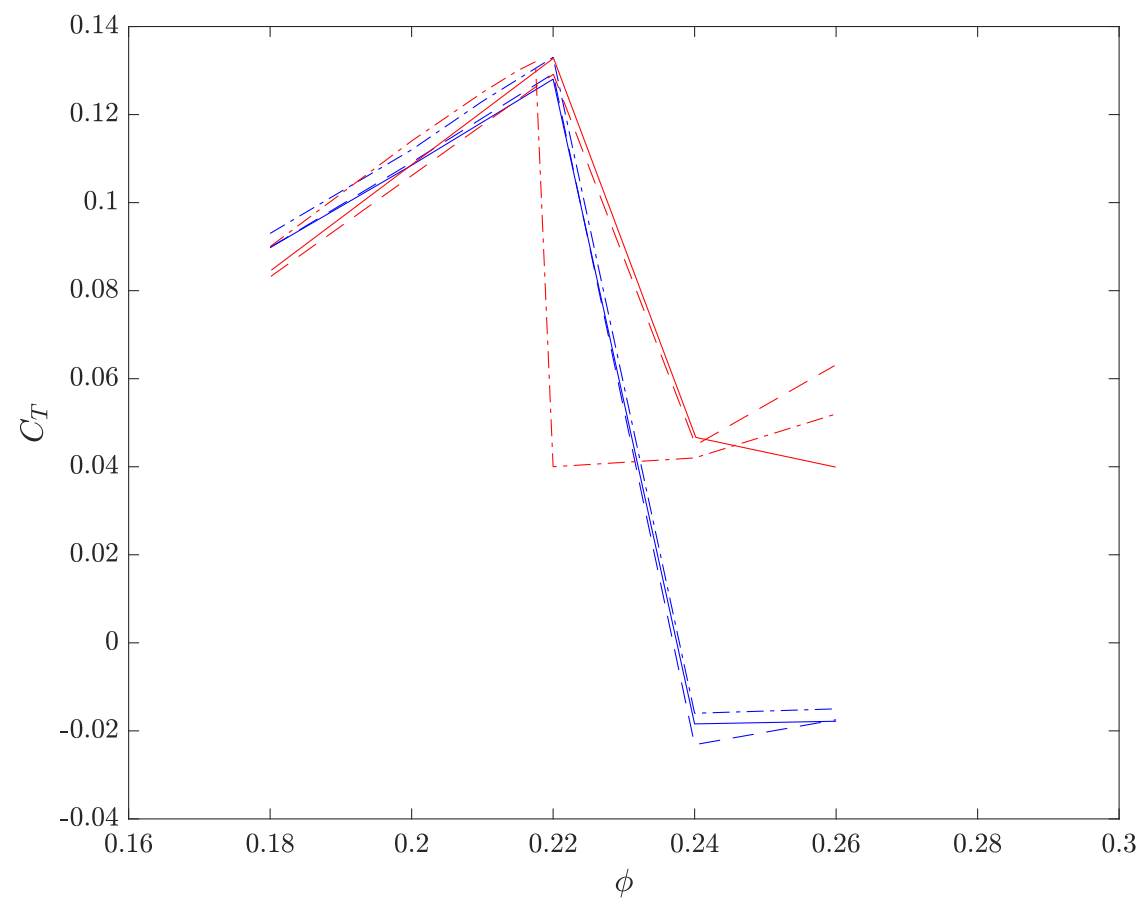

Figure 4: Mesh dependency studies 
good progress in a reasonable time frame, as the solver must converge for each evaluation of the objective function.

Experimental data typically shows a hysteretic loop between the acceleration and deceleration of the flow [38]. The origin of the hysteresis has been explained with the air compressibility in the OWC chamber [24, 36, 39]. This is, however, an unsteady feature of the system rather than of the turbine (where dynamic effects are negligible [40]), and it will not be considered in this work. In fact, the turbine operates at a reduced frequency $\left(k=\pi f c / U_{\infty}\right)$ of $2 \times 10^{-4}$, well below what would be considered to produce noticeable dynamic effects [41]. Therefore, given that the Mach number based on the tip blade speed is 0.15 , the incompressible assumption is considered acceptable.

Gauss integration was used for all flux calculations. Gradients and Laplacian terms were discretised with a second-order central differencing scheme, while convective terms were discretised with a second-order scheme with a flexible limiter acting in regions of rapidly changing gradients (limited Gauss in OpenFoam). The under-relaxation factors were fixed to 0.5 for pressure and velocity, 0.6 for all other quantities.

The chord-based Reynolds number at the tip is $\sim 10^{5}$, suggesting that turbulent transition could be present. A $k-k l-\omega$ transition algorithm was the only verified transition model available in OpenFOAM. However, a better fit to the experimental data was found using the $k-\omega$ shear stress transport model [42]. The mesh was generated ensuring that the maximum $y^{+}$value on all the walls was of the order of 1 , in order to accurately solve the boundary layer down to the wall, without the need for wall-functions.

A moving reference frame (MRFProperties dictionary in OpenFoam) was used to represent the rotor motion. The rotor and a portion of the duct wall extending to 0.5 chord lengths upstream and downstream of the blade was considered in motion. Flow velocity (with the value appropriate to generate the desired flow coefficient) and zero pressure gradient were set at the inlet to the domain, and a uniform pressure and zero normal velocity gradient were used at the exit. All walls had a no-slip condition. Values of $0.135 \mathrm{~m}^{2} \mathrm{~s}^{-2}$ and 300 
$\mathrm{s}^{-1}$ have been used for $k$ and $\omega$, respectively. These correspond to a turbulence intensity of $3 \%$ and a length scale of $2 \mathrm{~mm}$.

The convergence was assessed by monitoring the torque coefficient and axial force coefficient. Evaluations were terminated when the differences between the maximum and minimum values of both were less than $1 \%$ of the mean over the last 200 iterations. An average of 1500 iterations were required for convergence. Simulations that did not converge after 3000 iterations were stopped, and the blade was considered stalled.
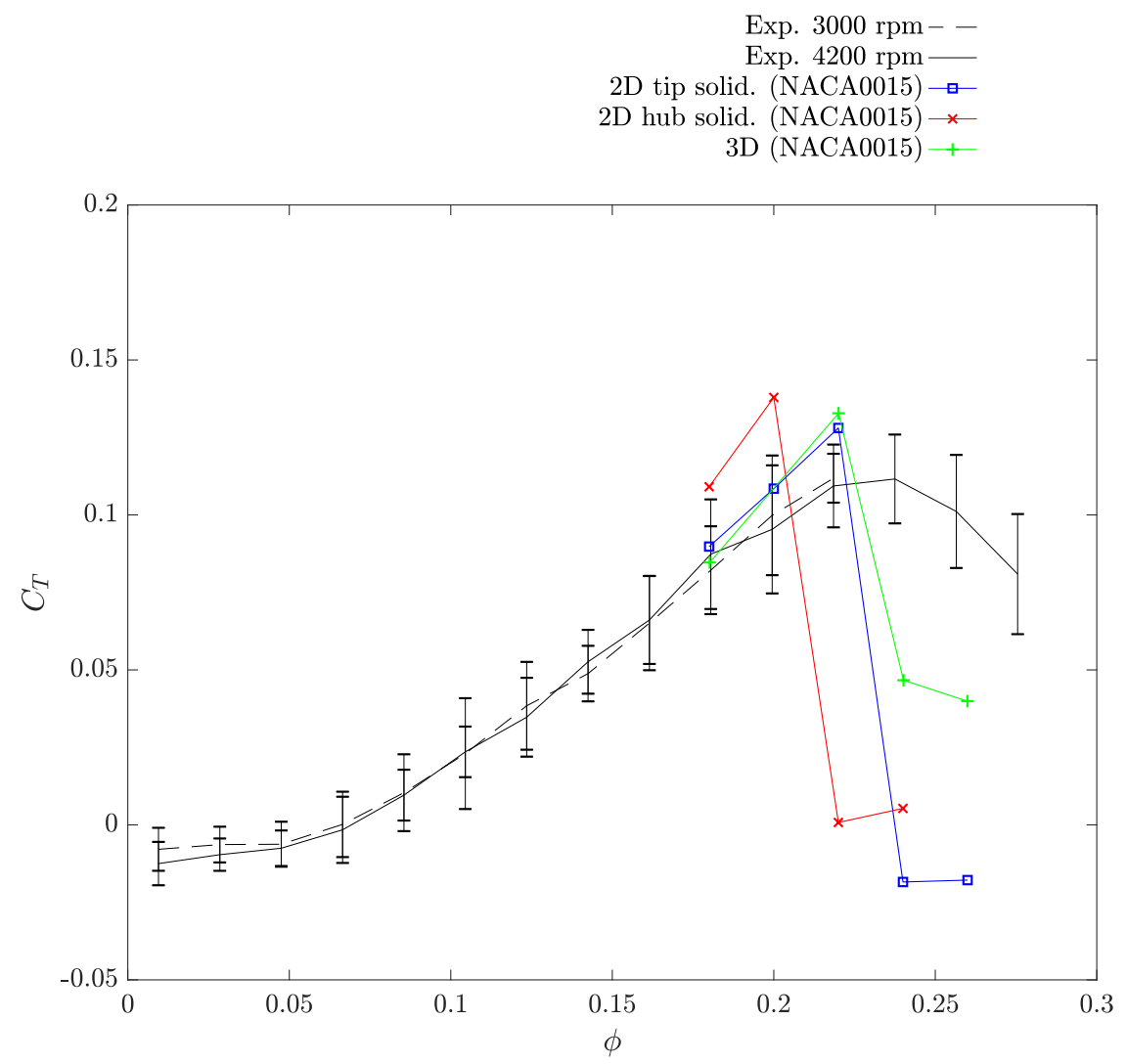

Figure 5: Torque coefficient plotted against flow coefficient for the 2D and 3D CFD with reference experimental data from [35]

With these settings, the variation in torque coefficient with flow coefficient was plotted in Figure 5, which also shows experimental data for an identical turbine at two rotational speeds [35], the one used in this work and a smaller one, 
which leads to a larger operating range. As a Wells turbine operates with a low subsonic flow (in the incompressible range), aerodynamic performance curves, when represented in non-dimensional terms, are a function of only the Reynolds number. Moreover, this being a weak dependency within the range spanned in the experiments [35, 43], the expected variation in performance due to changes in rotational speed is minor [44], certainly lower than the uncertainty due to experimental measurement. Experimental performance curves are derived using measured flow velocities in the vicinity of the blade to avoid inconsistencies introduced when neglecting the capacitive delays caused by experimental setups [38]: error bars due to the measurement of the local flow velocity with hotwire probes are reported. Other uncertainties (for example those due to tip gap variability [37]) are difficult to consider, but could have an effect on the measured performance.

For the $2 \mathrm{D}$ mesh, the torque coefficient has been calculated by assuming a uniform force along the blade. The evaluation over-predicts the torque coefficient but does accurately capture the stall point, at a flow coefficient of 0.22 for the $2 \mathrm{D}$ tip solidity and 3D cases, and 0.20 for the 2D hub solidity case. At higher flow coefficients, a steady solution could not be obtained, so the results in this region have to be considered with caution.

A more detailed validation and analysis of the flow field in this turbine has been presented in 24, 39], while different geometries and operating conditions were studied in [36, 45]. The interested reader is referred to these works for in-depth analyses of the impact of turbulence closure and dynamic operating conditions on Wells turbine performance. Regarding the former, the $k-\omega$ model appears a good choice to predict the performance with attached flow and the occurrence of stall, while other turbulence models [46] can be more appropriate in the prediction of the performance under deep-stall conditions. However, this is a condition to be avoided in turbomachinery operation as it causes a significant drop in performance and mechanical issues. For these reasons, the $k-\omega$ model is deemed appropriate for the current work. Regarding the latter, in [36, 39, 47], it is shown how dynamic effects in a Wells turbine are negligible, 
and the hysteresis [38, 48], previously attributed to turbine aerodynamics, is in reality a hysteresis of the whole OWC system, caused by the capacitive behavior of the chamber. Hence, no difference in performance is expected between steady and dynamic operating conditions.

In light of the above considerations, and of the renowned ability of CFD to predict relative changes better than absolute values [49], the described simulation system is considered satisfactory for the purpose of estimating relative changes in performance for a Wells turbine, which is the key aspect of an optimization study.

\section{Two-dimensional optimization}

\subsection{Flow coefficient}

Given that the Wells turbine only provides useful power output at high flow

coefficients close to stall [50], it is intuitive that the optimization should be performed in this region. An initial run was done at a flow coefficient of 0.18. While this gave good improvements, increasing the torque coefficient by $54 \%$ when compared to the NACA0015 blade at the same flow coefficient, Figure 6 shows that the stall point has also moved to the lower flow coefficient, decreasing the operating range of the machine where power generation will be possible. When compared to the NACA0015 blade at its peak torque coefficient, the improvement is only $7 \%$. This means that the OWC would need to be modified in order to give a suitable range of flow coefficients for the improvement to be realised in an increased power output. This is not something that this investigation considered, so it was concluded that optimizations should be run at the highest flow coefficient for which the original profile can retain attached flow. For all the following optimizations, a flow coefficient of 0.22 was therefore chosen for the $3 \mathrm{D}$ and $2 \mathrm{D}$ tip solidity meshes, while a value of 0.20 was chosen for the 2D hub solidity meshes (see Figure 5 for stall points).

\subsection{Free form deformation control points}

As more control points are introduced to the FFD tool, the profile can be manipulated in a finer way. This also has the undesirable effect of increasing 


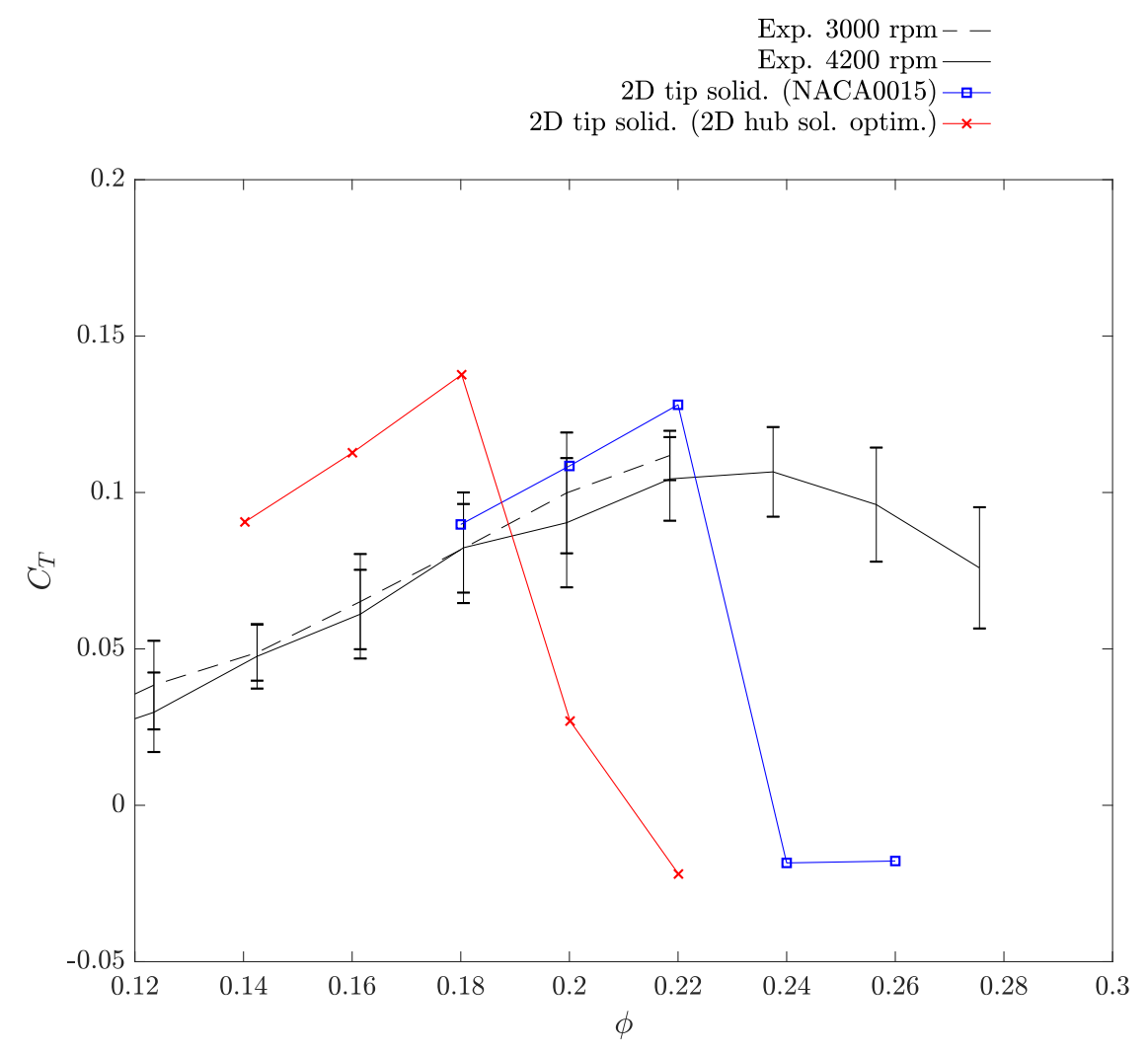

Figure 6: Torque coefficient plotted against flow coefficient after optimization at a flow coefficient of 0.18 
the size of the search space available to the tabu search, which would require more iterations and objective function evaluations to navigate towards the minimum. Trial optimizations were performed using 4, 8 and 12 control variables, corresponding to 2, 4 and 6 moveable control points at upper and lower regions of the grid.

All three optimizations were performed at the tip solidity with the same initial step size to the tabu search, and allowed a total of 200 iterations. The results in terms of two measures of computational cost are shown in Figure7 The 4 control variable case was able to make more progress as the step size was reduced towards the end of the optimization. However, for both measures of computational cost, the 8 control variable case produced the greatest improvement to the objective function in the least time. The 12 control variable case made slow progress initially and was unable to reach the same level of improvement due to the large search space, thus 8 control variables were chosen to manipulate each profile in the optimizations that follow.
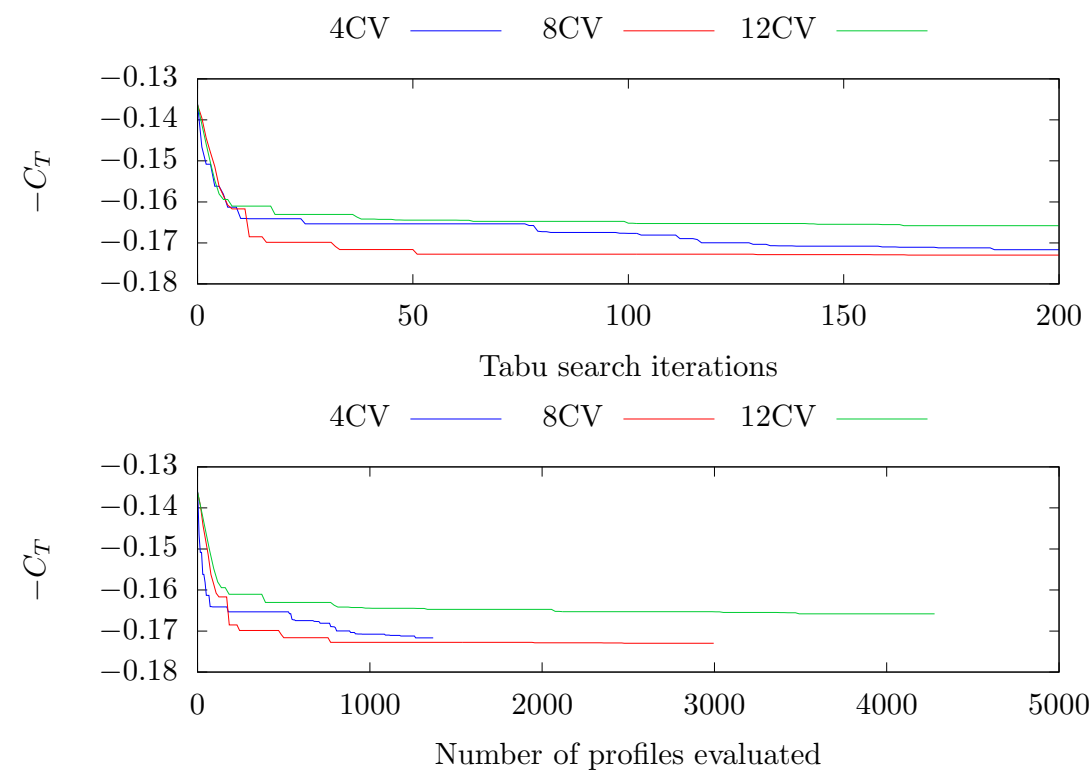

Figure 7: The progression of the tabu search for different numbers of control variables 
The profiles at the end of each optimization are shown in Figure 8, Each of the tabu searches progressed towards a similar profile, moving the point of maximum thickness forwards and narrowing the profile aft of this point.
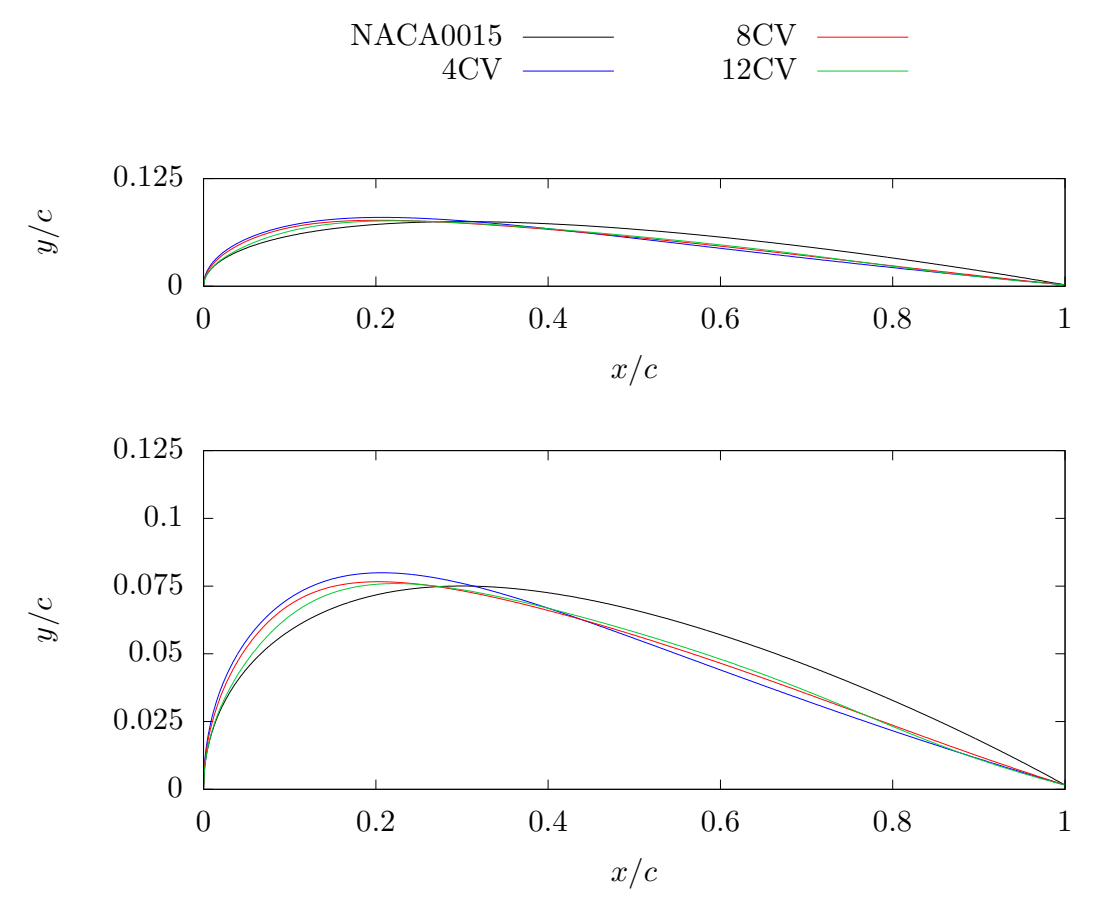

Figure 8: The half profiles at termination of the tabu search for different numbers of control variables

\subsection{Tip and hub solidity profile optimizations}

As the datum blade was built with a constant chord, the solidity varies linearly between a value of 0.844 at the hub and a value of 0.642 at the tip. To take into account the effect of the different solidities on the performance at different radial positions two optimizations were run, with the ultimate aim of investigating the performance of a $3 \mathrm{D}$ blade linearly varying between the two optimized profiles for hub and tip solidities.

After 100 iterations at the tip solidity, 1281 profiles had been evaluated in roughly 3 days on 17 cores. Figure 9 shows the initial NACA0015 and final 
profile. The torque coefficient had been increased by $34 \%$ compared to the value for the NACA0015 profile while the efficiency had decreased by $0.84 \%$. The improvement in torque coefficient was much larger than the $12 \%$ found in previous research [16], while the change in efficiency is found to be similarly small. In [16], a spline fit between 12 control points was used to represent the blade shape, amounting to 24 control variables. As was found in Section 3.2 , increasing the number of control variables above a critical value decreases the improvement that the tabu search is able to make, due to the increase in the size and complexity of the search space. This is suggested to be the main reason for the greater improvements that have been found, with smaller contributions from the greater number of profiles that are evaluated and the single-objective optimization that has been used. Figure 10 shows that the relative improvement was maintained at flow coefficients below the value of 0.22 at which the optimization was performed and that there has been no change to the stall angle.

At the hub solidity, a $32 \%$ improvement was made to the torque coefficient. The efficiency, however, decreased by $12 \%$. The blade shape at this point was unlike conventional aerofoils with a deeply concave profile near the midpoint, shown in Figure 9. The pressure coefficients on the airfoil in Figure 11] show a larger increase in the pressure difference at hub solidity than tip solidity. This explains the lower efficiency, as the pressure difference will cause a large increase in axial force. On the suction surface, the maximum adverse pressure gradient at the leading edge had become smaller and there is little increase to the adverse pressure gradient as the trailing edge is approached. This is key to avoiding boundary layer separation and stall.

Figure 12 plots torque coefficient against efficiency for all evaluated profiles that resulted in a torque coefficient larger than 0.12 (the value given by the datum profile) for both optimization runs (at the tip and hub solidities). The trade-off between the two figures of merit is evident. At the tip solidity, a profile was found that increased the efficiency by $1.8 \%$; however, the corresponding increase in torque coefficient was only $1.7 \%$. At the hub solidity, efficiency could 
NACA0015

Hub solidity

Tip solidity
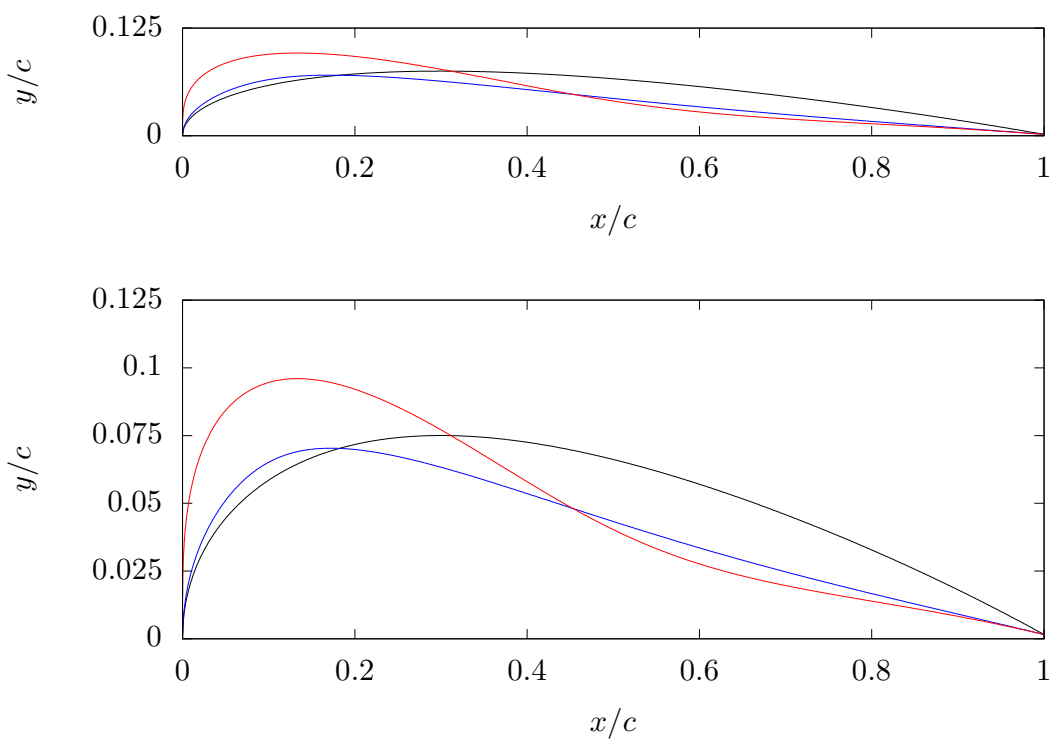

Figure 9: The half profiles of the blades optimized at hub and tip solidity

be improved by $1.4 \%$ but at the cost of a $3.3 \%$ reduction in the torque coefficient. This is consistent with a stronger correlation between a decrease in efficiency for an increasing torque coefficient at hub solidity. Therefore, at the tip solidity, the profile with the maximum torque coefficient should be chosen, whereas at the hub solidity, more careful consideration of the maximum allowable efficiency penalty may be required. Alternatively, a multi-objective optimization could be used to obtain a better trade-off. 
Exp. 3000 rpm - - -

Exp. $4200 \mathrm{rpm}$

$2 \mathrm{D}$ tip solid. (NACA0015) $\square$

$2 \mathrm{D}$ tip solid. (2D tip sol. optim.) $\longrightarrow$

2D hub solid. (NACA0015) -

2D hub solid. (2D hub sol. optim.) $\triangle \triangle$

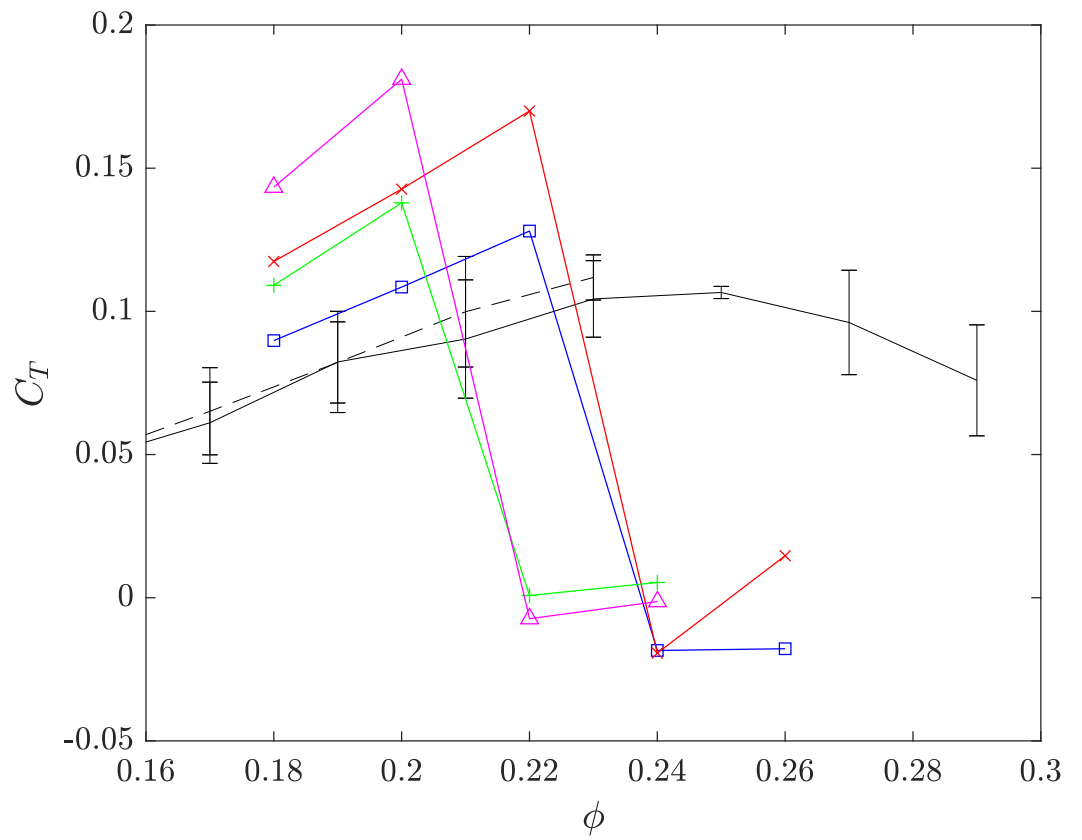

Figure 10: Torque coefficient plotted against flow coefficient for the 2D optimizations 
Tip solidity

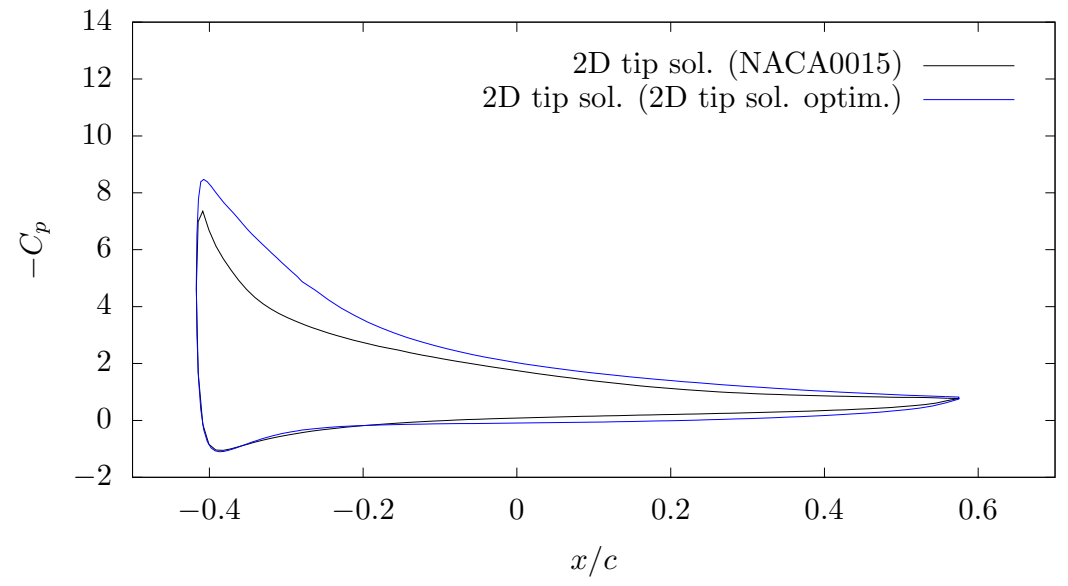

Hub solidity

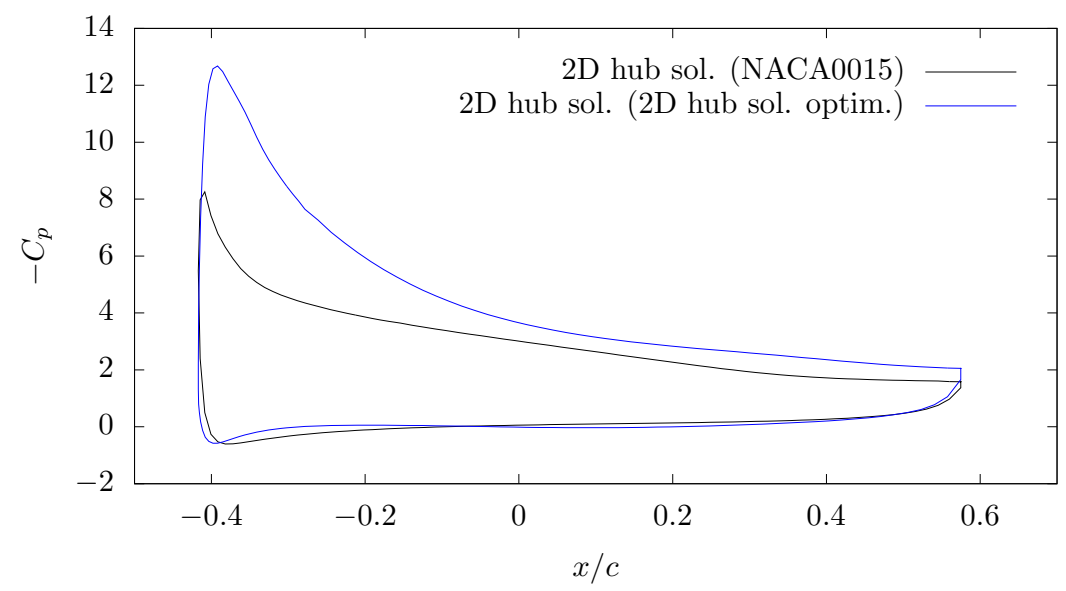

Figure 11: Plots of the surface pressure coefficient comparing the NACA0015 profile to the optimized profiles 
Tip solidity

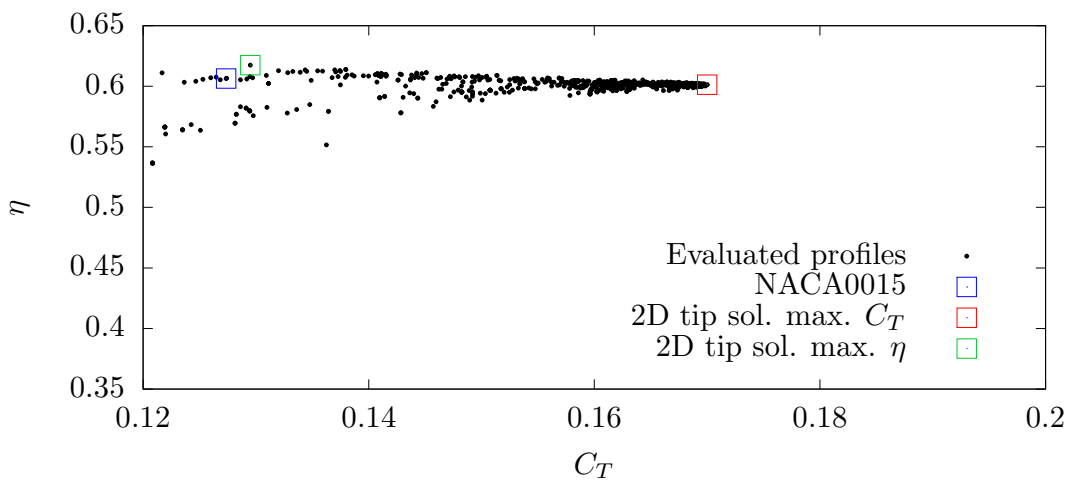

Hub solidity

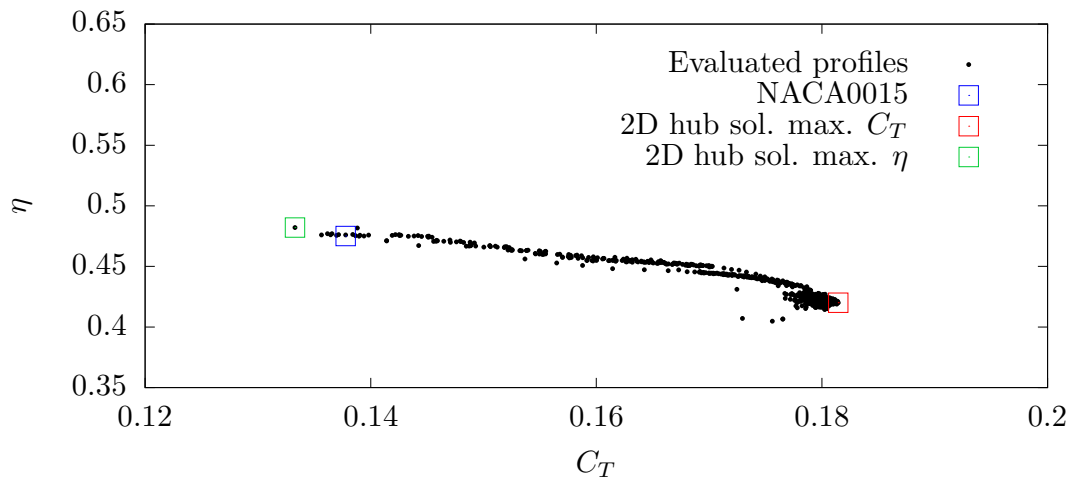

Figure 12: Scatter graph of efficiency against torque coefficient for all evaluated profiles that resulted in a high torque coefficient 


\section{Application of two-dimensional results to the three-dimensional turbine}

When the 3D geometry is generated by extruding the profile optimized at tip solidity, there is a $12 \%$ improvement to the torque coefficient, shown in Figure 13. This is much lower than was found in two dimensions. Here, torque coefficients are reported for the datum profile (experimental and numerical) and for the optimized blade at several flow coefficients near the one used for the optimizations. In 2D, the torque coefficient is calculated by assuming a constant force along the height of the blade. Therefore, if the same $34 \%$ increase was to be seen in 3D, an average increase of $34 \%$ to the local tangential force would be required along the entire length of the blade. It would be reasonable to assume that this would not be the case for the uniform blade profile, especially given the difference in the optimized profile between the tip and hub solidities. Figures 14 and 15 report pressure coefficients at three radial positions $(80 \%$, $50 \%$ and $20 \%$ of the span) on the blade and show that a greater suction peak towards the leading edge of the extruded optimised profile is the driving force for the increase in torque coefficient, while the adverse pressure gradients remain the same towards the rear, preventing early stall. It is also important to note that the maximum adverse pressure gradient is not larger than before.

Using a linear profile variation between the hub and tip optimized profiles improves the torque coefficient by a further 2 percentage points, to $14 \%$ above the NACA0015 blade. Figure 14] shows that this is achieved by further increasing the pressure difference across the blade as the hub is approached. There is clearly some reason that either the $2 \mathrm{D}$ optimization does not apply to the $3 \mathrm{D}$ case particularly well, or that there is a smaller potential for improvements in the $3 \mathrm{D}$ case. The surface pressure coefficients for the optimized profiles in $2 \mathrm{D}$ and linearly varying 3D blade are shown in Figure 16 with the NACA0015 data for reference. At the tip solidity, the $2 \mathrm{D}$ simulation is a good approximation to the $3 \mathrm{D}$ flow, indicating that the blade is contributing close to the predicted $34 \%$ improvement in this region. At the hub, the 2D surface pressure distribution is a poor representation of the $3 \mathrm{D}$ solution. There is a much smaller pressure 
Exp. $3000 \mathrm{rpm}---$

Exp. $4200 \mathrm{rpm} \longrightarrow$

$3 \mathrm{D}(\mathrm{NACA0015)} \square$

$3 \mathrm{D}(2 \mathrm{D}$ tip sol. optim.) $-\times$

$3 \mathrm{D}(2 \mathrm{D}$ hub+tip sol. optim) -1

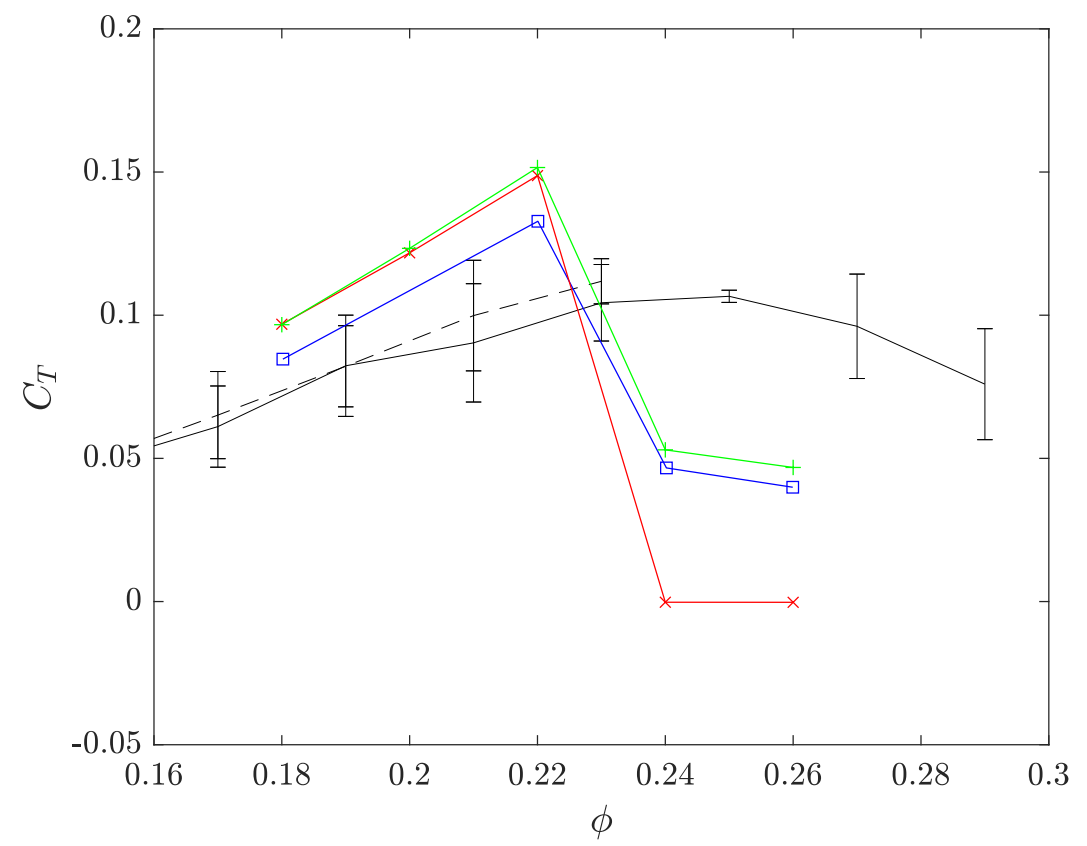

Figure 13: Torque coefficient plotted against flow coefficient for the 2D optimization results applied to $3 \mathrm{D}$ 

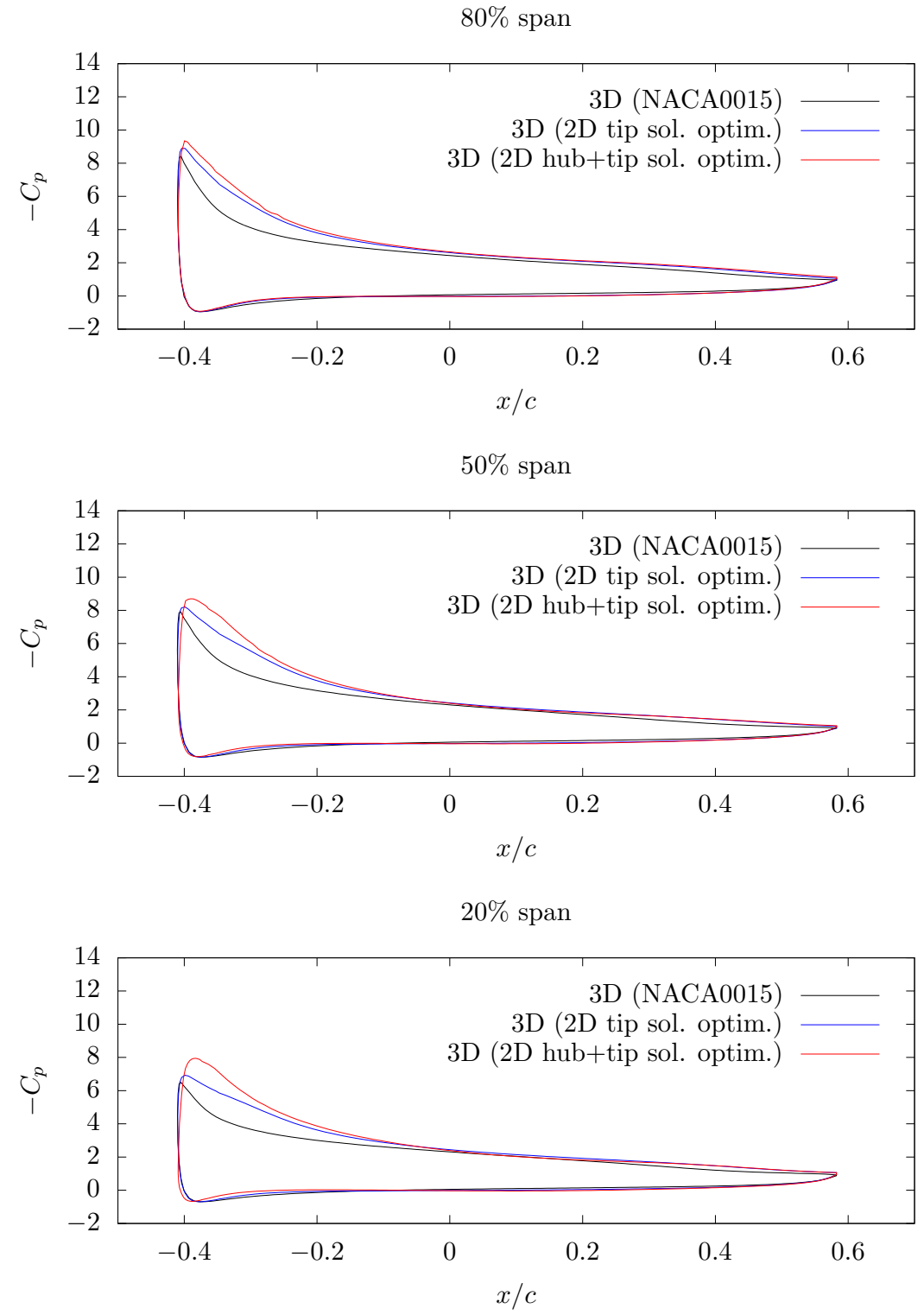

Figure 14: Plots of the surface pressure for the 2D optimization results applied to 3D 


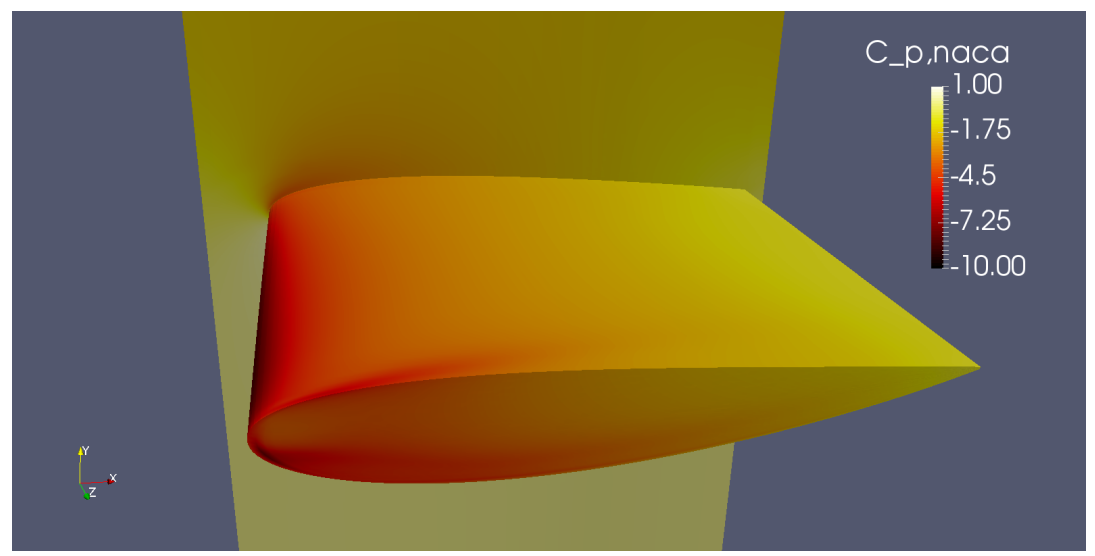

(a) NACA0015

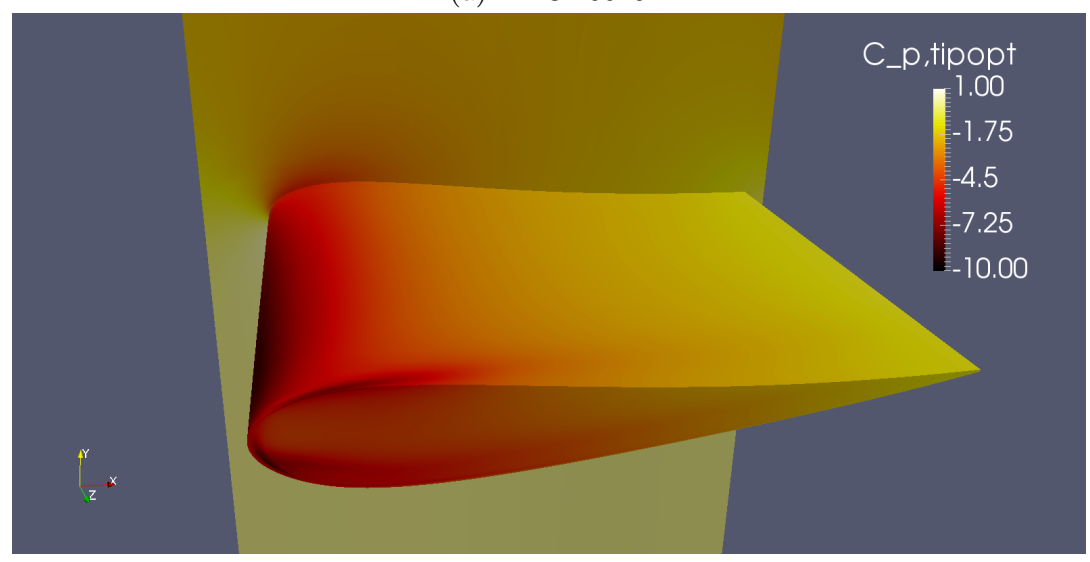

(b) Tip solidity optimized profile extruded along blade

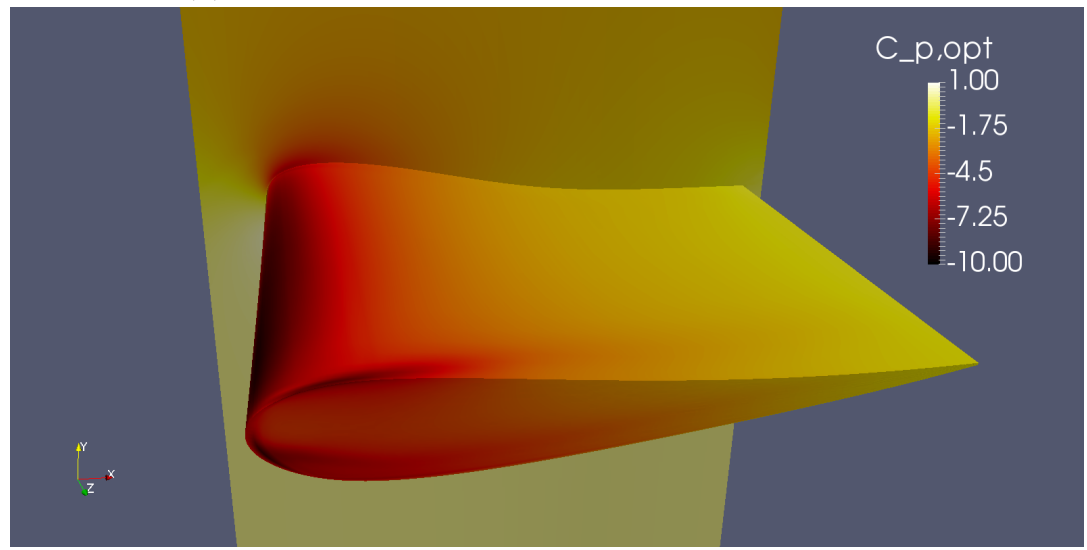

(c) Linearly varying between the hub and tip solidity optimized profiles

Figure 15: Pressure coefficients on the blade surface for each of the three-dimensional blades 
difference between the upper and lower surfaces in 3D and the local increase in the torque coefficient is likely to be much lower than the $32 \%$ predicted in $2 \mathrm{D}$. It is therefore possible to conclude that it is a smaller than predicted increase in local tangential force at the hub of the blade that causes the smaller relative increase in torque coefficient in $3 \mathrm{D}$ compared to the two $2 \mathrm{D}$ optimizations.
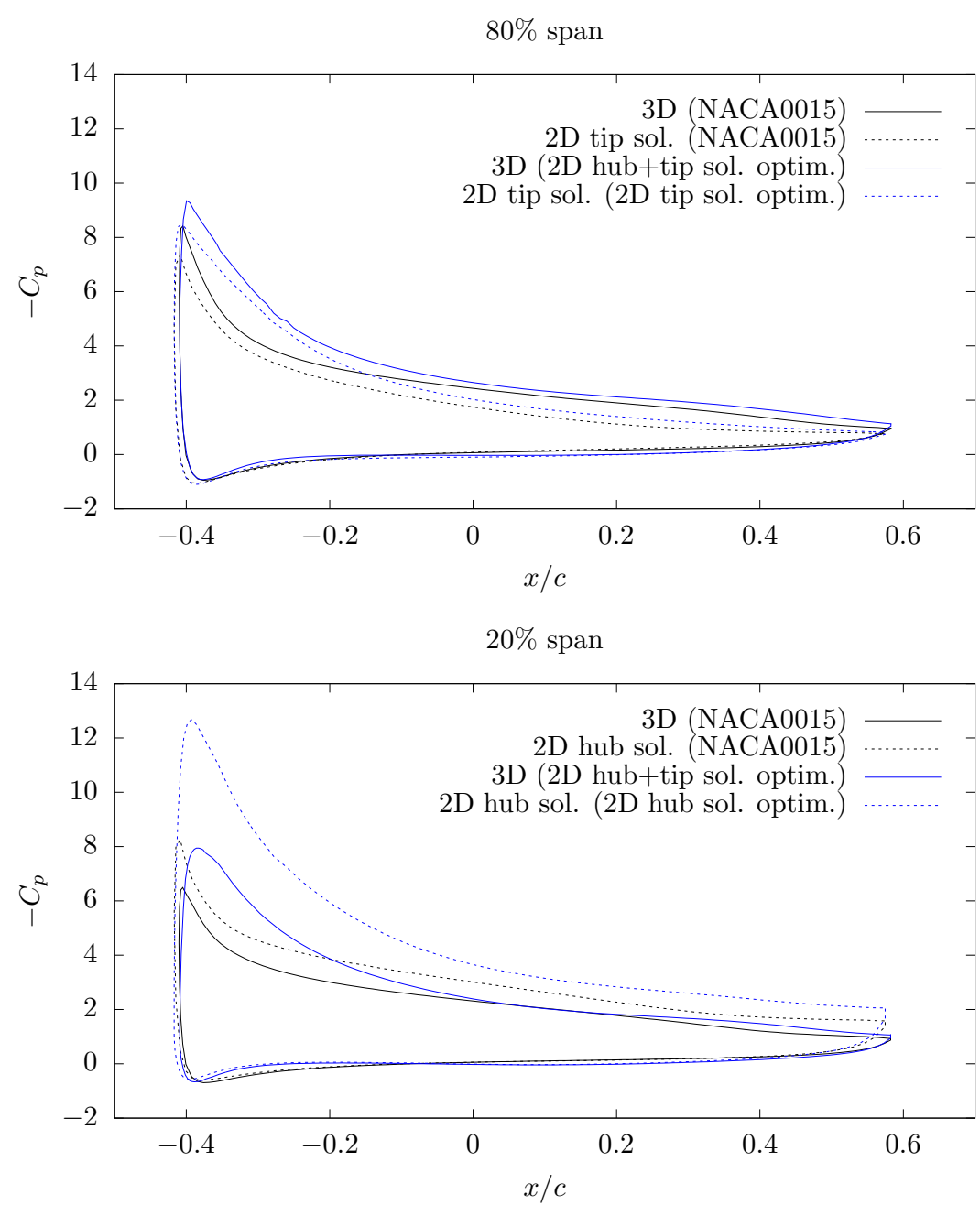

Figure 16: Plots comparing the 2D surface pressure coefficients to $3 \mathrm{D}$

Plotting the axial flow velocity on the periodic plane, midway between 
blades, against the radial position in Figure [17, a significant over-prediction of the local value is found in $2 \mathrm{D}$ at the hub compared to the $3 \mathrm{D}$ solution. This is due to the high solidity causing a significant blockage effect. This corresponds to a lower local flow coefficient for the 3D blade. Meanwhile, at the tip there is good agreement between the $2 \mathrm{D}$ and $3 \mathrm{D}$ results, especially as the $70 \%$ span position is approached. For the optimized blade profiles in $2 \mathrm{D}$, the predicted inlet pressure at the hub is 2.3 times higher than the inlet pressure at the tip for an equal inlet flow speed. As the solution is expanded to three dimensions, there is no physical means by which such a radial pressure gradient could be sustained, and an equilibrium is formed with air moving radially outwards as the blade is approached. In 3D, flow also moves through the tip gap, decreasing the axial velocity at the hub and increasing it at the tip [51]. The volume of air that moves radially outwards from the hub at inlet is roughly the same as the volume that flows through the tip gap, making the $2 \mathrm{D}$ tip solidity flow conditions a good approximation to the 3D conditions.

Therefore, in order to attempt to realise the greatest possible improvements in $3 \mathrm{D}$ by optimising in $2 \mathrm{D}$, the $2 \mathrm{D}$ inlet axial flow should be adjusted so that the local flow conditions at the blade match the 3D case. This would require a reduction to the axial inlet velocity in $2 \mathrm{D}$ at the hub, while increasing it slightly at the tip, meaning that the axial flow velocity at inlet to the hub solidity mesh is lower than the tip solidity mesh. There is no radial variation to the inlet axial flow in 3D; however, the key difference is that there can be no radial component to the flow velocity from inlet to blade in the $2 \mathrm{D}$ mesh while there can be in 3D. This effectively fixes the volumetric flow rate for all axial positions in the 2D mesh. The volumetric flow rate near the blade through a slice of the 3D mesh at the solidity of interest should be calculated, and referred back to the inlet of the respective 2D mesh in order to make the local flow conditions at the blade equal in 2D and 3D. These boundary conditions for velocity should then be used when optimising the profiles in $2 \mathrm{D}$. It is predicted that there will still be a difference in the local flow coefficient when the two optimized profiles are used in 3D due to the increased blade loading changing this effect slightly; however, 
3D (2D hub+tip sol. optim.)

$2 \mathrm{D}$ tip sol. (2D tip sol. optim.)

$2 \mathrm{D}$ hub sol. (2D hub sol. optim.)

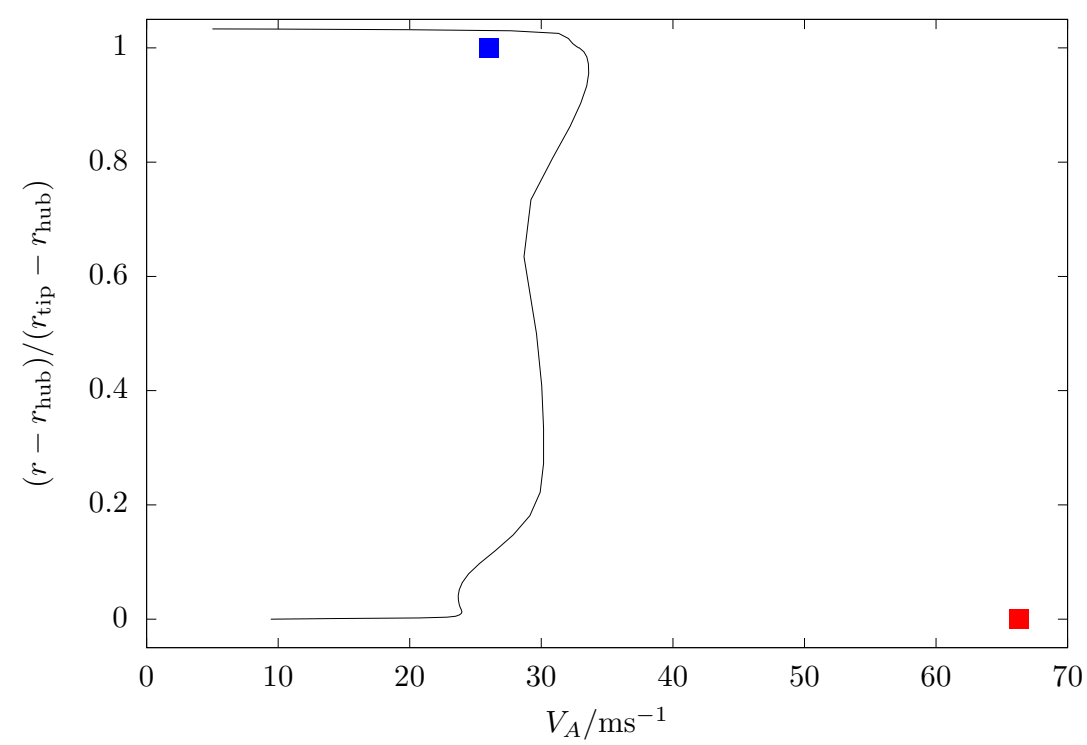

Figure 17: Plots of the axial flow velocity profile midway between blades against radius for the optimized profile

the magnitude of error should be substantially lower, increasing the extent to which the 2D improvements represent the improvements in 3D. Given that large improvements of the order of $30 \%$ have been found in both 2D optimizations, an improvement of more than $14 \%$ in 3D should be possible using this method.

The efficiency of the turbine with the linearly varying profile is $7.2 \%$ lower than the NACA0015 turbine, due to an increase in the axial force coefficient, evident by the greater pressure drop across the blade shown in Figure 19. This value is between the two values that were predicted in $2 \mathrm{D}$. It has been found that the tip gap effects can have a large impact on the efficiency of the turbine [37]. Figure 18 shows plots of the axial flow velocity in this region for the NACA0015 and linearly varying profile. The mean and peak flow speeds through the tip gap are higher for the optimized profile and can explain some of the efficiency decrease. The greater pressure difference between the upper and lower surfaces 
especially near the leading edge, as shown in Figure 14, is the driving force for this difference. This means that, as the profile is improved, methods of preventing the tip leakage flow, such as end plates [52] or grooves [53, 54], will become more important.

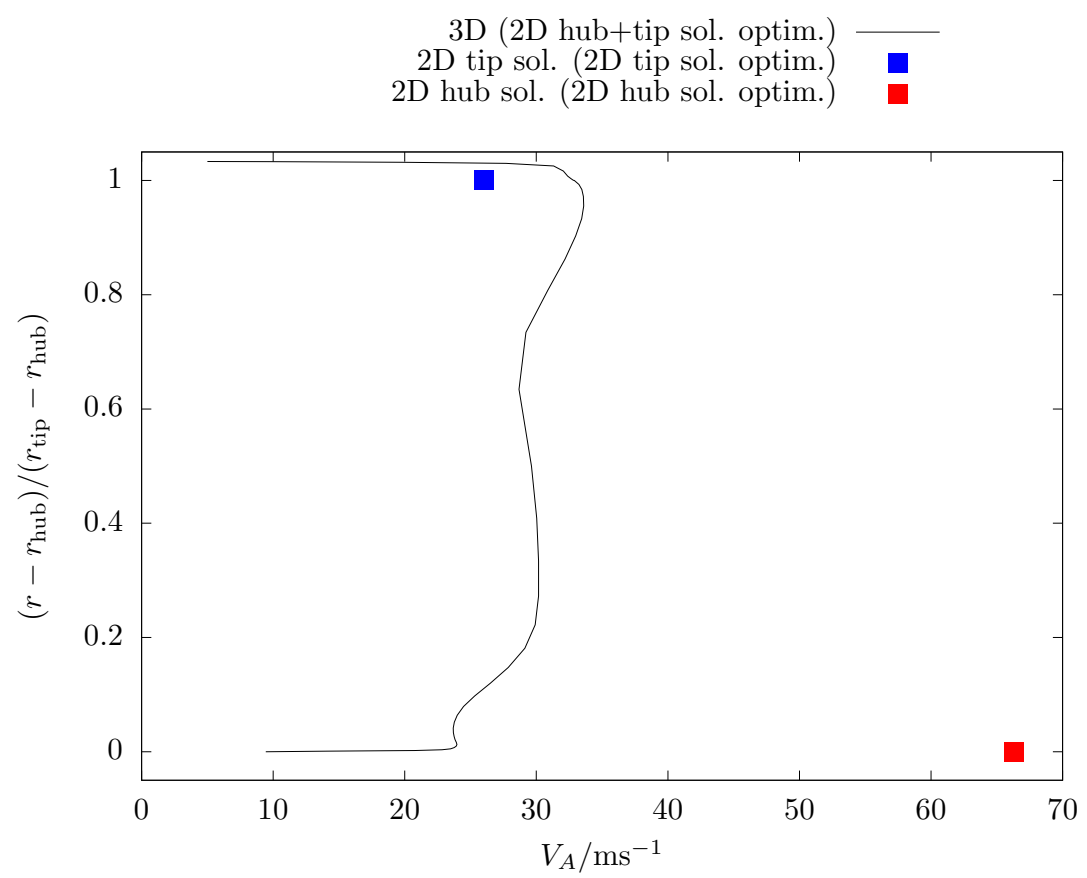

Figure 18: Plots of the axial flow velocity in the tip gap region against tangential position with the blade position indicated 


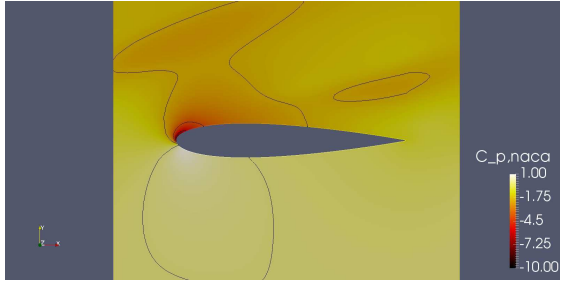

(a) $80 \%$ span, NACA0015 profile

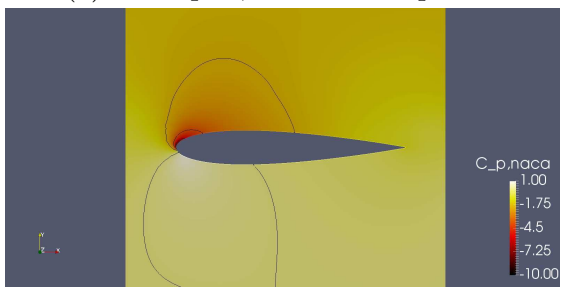

(c) $50 \%$ span, NACA0015 profile

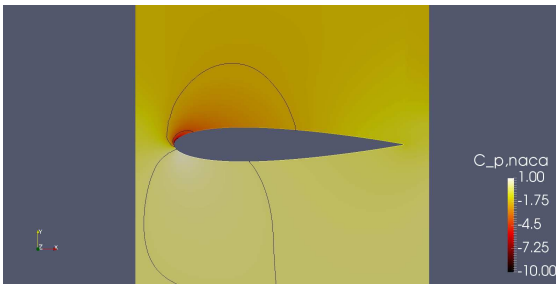

(e) $20 \%$ span, NACA0015 profile

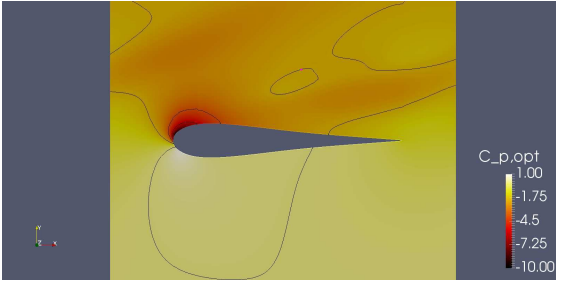

(b) $80 \%$ span, linearly varying profile

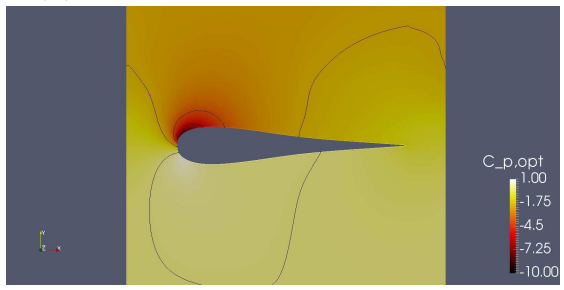

(d) $50 \%$ span, linearly varying profile

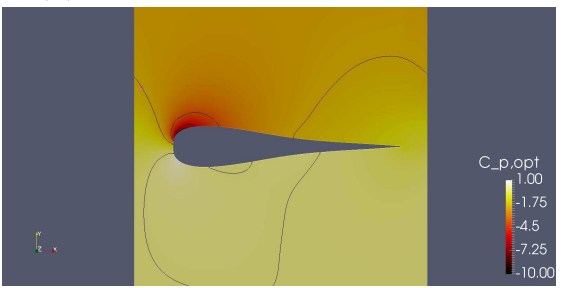

(f) $20 \%$ span, linearly varying profile

Figure 19: Colour maps and contours of the pressure coefficient at different radial positions (colour scales and contour levels have been kept the same between plots)

\section{Conclusions}

An investigation into the optimization of the Wells turbine is presented in this paper. A method for simulating the flow in the turbine using the open source software OpenFOAM was developed and linked to an in-house tabu search optimization algorithm with the aim of increasing the performance of the turbine by modifying the blade profile using an in-house free form deformation tool. In an attempt to reduce the computational cost of this process, the results of two 2D optimizations have been applied to 3D and the differences assessed. The following conclusions can be drawn:

- An optimized profile will be on the limit of stall at the flow coefficient for which the objective function evaluation took place, as this gives the great- 
est improvements to performance. Therefore, if the stall performance of the original profile is to be retained in the optimized blade, the evaluation should take place at the highest flow coefficient for which the flow can remain attached in the original design. The optimized profile for a high flow coefficient will also improve performance at lower flow coefficients.

- Using a free form deformation method to manipulate the profile allowed for good improvements with a limited number of control variables. There was an optimal number of control variables for which the profile could be manipulated accurately while also keeping the search space available to the tabu search as small as possible. The proposed approach allowed improvements significantly larger than those obtained by previous research focusing on blade profile modification, thank to the more effective representation (and lower complexity) of the search space.

- The optimized profile depended strongly on the radial location and therefore solidity at which the optimization took place. The relative increases in the torque coefficient at the tip and hub were $34 \%$ and $32 \%$ respectively. At the hub solidity, there was a stronger negative correlation between the efficiency and the torque coefficient. At the tip solidity, the efficiency was almost constant for large changes in torque coefficient.

- When the two optimized profiles were applied to the 3D mesh, the relative increase in torque coefficient of $14 \%$ was much smaller than was predicted in $2 \mathrm{D}$. This was primarily due to the $2 \mathrm{D}$ hub solidity simulation being a poor representation of the local flow conditions at the hub in $3 \mathrm{D}$. The local flow coefficient at the hub in $3 \mathrm{D}$ is much lower than that found by applying a uniform axial inlet velocity to the two $2 \mathrm{D}$ meshes. The mass of fluid moving through the tip gap region is approximately equal to the decrease in flow at the hub, making the 2D tip solidity simulation a good approximation to the local flow conditions at the tip of the $3 \mathrm{D}$ simulation. Previously optimized profiles for the Wells turbine had not been verified in $3 \mathrm{D}$, and it is therefore reasonable to assume that a similar mismatch 
(or reduction in the performance improvements obtained) could have been observed.

- It is proposed that the inlet axial flow velocity to the $2 \mathrm{D}$ simulations should be adjusted so that the flow conditions near the blade match the local flow conditions near the blade in 3D for the original profile. Then, if two optimizations were to be run using these boundary conditions, the relative increases in $2 \mathrm{D}$ would be more representative of the relative increase in $3 \mathrm{D}$. The efficiency is likely to be lower in 3D due to the increased pressure difference between the blade surfaces driving more flow through the tip gap.

- Using the 3D simulation as the objective function evaluation for the optimization algorithm increases the computational cost to a level where sufficient progress could not be made in a reasonable time frame. This is not only due to the larger mesh size increasing the clock time for each tabu search iteration, but also due the increase in the number of control variables. Steady simulations have been used to drive the optimization process, but dynamic effects are expected to be negligible, at least from the point of view of the turbine. Recent studies [24, 36, 39, 47] have shown how the well-known Wells turbine hysteresis [38] is, in fact, not due to an aerodynamic hysteresis of the turbine, negligible at the non-dimensional frequency it operates at, but to the capacitive behavior of the OWC system.

\section{Acknowledgements}

This work has been funded by the Regione Autonoma Sardegna under grant F72F16002880002 (L.R. 7/2007 n. 7 - year 2015). 


$\begin{array}{ll}\text { Nomenclature } \\ c & \text { Blade chord } \\ c_{p} & \text { Pressure coefficient } \\ C_{A} & \text { Axial force coefficient } \\ C_{T} & \text { Torque coefficient } \\ D & \text { Blade drag force } \\ f_{T} & \text { Blade tangential force per unit radius } \\ F_{A} & \text { Blade axial force } \\ F_{T} & \text { Blade tangential force } \\ L & \text { Blade lift force } \\ r & \text { Radius } \\ T & \text { Blade torque } \\ U & \text { Blade speed } \\ V & \text { Absolute flow velocity } \\ \eta & \text { Efficiency } \\ \Omega & \text { Blade angular velocity } \\ \phi & \text { Flow coefficient } \\ \rho & \text { Density of air }\end{array}$




\section{Definitions}

$$
\begin{aligned}
& \phi=\frac{V_{A, \text { inlet }}}{\Omega r_{\text {tip }}} \\
& T=\int_{r_{\text {hub }}}^{r_{\text {tip }}} r f_{T} d r \\
& C_{T}=\frac{T}{\frac{1}{2} \rho \Omega^{2} r_{\text {tip }}^{5}} \\
& C_{A}=\frac{\Delta P}{\frac{1}{2} \rho \Omega^{2} r_{\text {tip }}^{2}} \\
& \eta=\frac{C_{T}}{C_{A} \phi} \frac{1}{\pi\left(1-\frac{r_{h}^{2}}{r_{t}^{2}}\right)} \\
& C_{p}=\frac{p-p_{\text {inlet }}}{\frac{1}{2} \rho \Omega^{2} r_{\text {tip }}^{2}}
\end{aligned}
$$

\section{References}

[1] R. Pelc, P. Fujita, Renewable energy from the ocean, Marine Policy 26 (2002) 471-479. doi:10.1016/S0308-597X (02) 00045-3.

[2] A. Wells, Rotary transducers, US Patent 4,221,538 (September 1980).

[3] T. V. Heath, A review of oscillating water columns, Philosophical Transactions of the Royal Society of London A: Mathematical, Physical and Engineering Sciences 370 (1959) (2011) 235-245. doi:10.1098/rsta.2011.0164

[4] A. F. Falcao, J. C. Henriques, Oscillating-water-column wave energy converters and air turbines: A review, Renewable Energy 85 (2016) 1391-1424. doi:10.1016/j.renene.2015.07.086

[5] Y. Luo, Z. Wang, G. Peng, Y. Xiao, L. Zhai, X. Liu, Q. Zhang, Numerical simulation of a heave-only floating OWC (oscillating water column) device, Energy 76 (2014) 799-806. doi:10.1016/j.energy.2014.08.079. 
[6] I. Simonetti, L. Cappietti, H. Elsafti, H. Oumeraci, Optimization of the geometry and the turbine induced damping for fixed detached and asymmetric OWC devices: A numerical study, Energy 139 (2017) 1197-1209. doi:10.1016/j.energy.2017.08.033

[7] ANSYS, ANSYS Fluent Theory Guide, Canonsburg, Pennsylvania, USA, 15th Edition (November 2013).

[8] T. Kim, T. Setoguchi, K. Kaneko, S. Raghunathan, Numerical investigation on the effect of blade sweep on the performance of Wells turbine, Renewable Energy 25 (2002) 235-248.

[9] P. Halder, S. H. Rhee, A. Samad, Numerical optimisation of Wells turbine for wave energy extraction, International Journal of Naval Architecture and Ocean Engineering 9 (1) (2017) 11-24.

[10] ANSYS, ANSYS CFX Reference Guide, Canonsburg, Pennsylvania, USA, 15th Edition (November 2013).

[11] W. Dawes, The practical application of solution-adaptation to the numerical simulation of complex turbomachinery problems, Progress in Aerospace Sciences 29 (3) (1992) 221-269.

[12] J. Watterson, S. Raghunathan, Computed effects of solidity on Wells turbine performance, JSME International Journal, Series B 41 (1) (1998) 177183.

[13] R. Soltanmohamadi, E. Lakzian, Improved design of Wells turbine for wave energy conversion using entropy generation, Meccanica 51 (8) (2016) 17131722. doi:10.1007/s11012-015-0330-x.

[14] S. Shaaban, Wave energy harvesting using a novel turbine rotor geometry, International Journal of Energy Research 41 (4) (2017) 540-552.

[15] T. Setoguchi, M. Takao, K. Itakura, M. Mohammad, K. Kaneko, A. Thakker, Effect of rotor geometry on the performance of Wells turbine, 
in: Proceedings of the 13th International Offshore and Polar Engineering Conference (ISOPE'03), Vol. 1, Honolulu, Hawaii, USA, 2003, pp. 345-350.

[16] M. Mohamed, G. Janiga, E. Pap, D. Thevenin, Multi-objective optimization of the airfoil shape of Wells turbine used for wave energy conversion, Energy 36 (2011) 438-446.

[17] S. Shaaban, Wells turbine blade profile optimization for better wave energy capture, International Journal of Energy Research 41 (12) (2017) 17671780, eR-16-7511.R3. doi:10.1002/er.3745.

[18] F. R. Torres, P. R. F. Teixeira, E. Didier, Study of the turbine power output of an oscillating water column device by using a hydrodynamic - Aerodynamic coupled model, Ocean Engineering 125 (2016) 147-154. doi:10.1016/j.oceaneng.2016.08.014.

[19] I. Simonetti, L. Cappietti, H. Elsafti, H. Oumeraci, Evaluation of air compressibility effects on the performance of fixed OWC wave energy converters using CFD modelling, Renewable Energy 119 (2018) 741-753. doi:10.1016/j.renene.2017.12.027.

[20] B. Bouali, S. Larbi, Sequential optimization and performance prediction of an oscillating water column wave energy converter, Ocean Engineering 131 (May 2015) (2017) 162-173. doi:10.1016/j.oceaneng.2017.01.004.

[21] A. Elhanafi, G. Macfarlane, A. Fleming, Z. Leong, Experimental and numerical measurements of wave forces on a 3D offshore stationary OWC wave energy converter, Ocean Engineering 144 (2017) 98-117. doi:10.1016/j.oceaneng.2017.08.040.

[22] F. Mahnamfar, A. Altunkaynak, Comparison of numerical and experimental analyses for optimizing the geometry of OWC systems, Ocean Engineering 130 (2017) 10-24. doi:10.1016/j.oceaneng.2016.11.054

[23] F. Glover, Tabu search - Part I, ORSA Journal on Computing 1 (3) (1989) 190-206. 
[24] T. Ghisu, P. Puddu, F. Cambuli, Numerical analysis of a Wells turbine at different non-dimensional piston frequencies, Journal of Thermal Science 24 (6) (2015) 535-543.

[25] C. Greenshields, OpenFOAM - The open source CFD toolbox - User's guide, CFD Direct Ltd., Version 4.0 (June 2016).

[26] H. Weller, G. Tavor, H. Jasak, C. Fureby, A tensorial approach to computational continuum mechanics using object-oriented techniques, Computers in Physics 12 (6) (1998) 620-631.

[27] D. Jaeggi, G. Parks, T. Kipouros, P. Clarkson, The development of a multiobjective tabu search algorithm for continous optimisation problems, European Journal of Operational Research 185 (2008) 11921212.

[28] T. Ghisu, G. T. Parks, D. M. Jaeggi, J. P. Jarrett, P. J. Clarkson, The benefits of adaptive parametrization in multi-objective tabu search optimization, Engineering Optimization 42 (10) (2010) 959-981. doi:10.1080/03052150903564882.

[29] S. Shahpar, Automatic aerodynamic design optimisation of turbomachinery components - an industrial perspective, VKI Lecture Series on Optimisation Methods \& Tools for Multicriteria/Multidisciplinary Design (2004) $1-40$.

[30] W. Song, A. J. Jeane, A study of shape parameterisation methods for airfoil optimisation, in: 10th AIAA/ISSMO Multidisciplinary Analysis and Optimization Conference, no. AIAA 2004-4482, New York, USA, 2004.

[31] D. A. Masters, N. J. Taylor, T. Rendall, C. B. Allen, D. J. Poole, Review of Aerofoil Parameterisation Methods for Aerodynamic Shape Optimisation, in: 53rd AIAA Aerospace Sciences Meeting, 2015. doi:10.2514/6.2015-0761. 
[32] J. A. Samereh, Survey of shape parameterization techniques for highfidelity multidisciplinary shape optimization, AIAA Journal 39 (5) (2001) 877-883.

[33] T. W. Sederberg, S. Parry, Free-form deformation of solid geometric models, Computer Graphics 20 (4) (1984) 151-160.

[34] T. Ghisu, J. Jarrett, G. Parks, Robust design optimization of airfoils with respect to ice accretion, Journal of Aircraft 48 (1) (2011) 287-304. doi:10.2514/1.C031100.

[35] P. Puddu, M. Paderi, C. Manca, Aerodynamic characterization of a Wells turbine under bi-directional airflow, Energy Procedia 45 (2014) 278-287.

[36] T. Ghisu, P. Puddu, F. Cambuli, Physical Explanation of the Hysteresis in Wells Turbines: A Critical Reconsideration, Journal of Fluids Engineering, Transaction of the ASME 138 (11) (2016) 1-9. doi:10.1115/1.4033320.

[37] M. Torresi, S. Camporeale, P. Strippoli, G. Pascazio, Accurate numerical simulation of a high solidity Wells turbine, Renewable Energy 33 (2008) 735-747.

[38] T. Setoguchi, M. Takao, K. Kaneko, Hysteresis on Wells turbine characteristics in reciprocating flow, International Journal of Rotating Machinery 4 (1) (1998) 17-24. doi:10.1155/S1023621X98000025.

[39] T. Ghisu, P. Puddu, F. Cambuli, A detailed analysis of the unsteady flow within a Wells turbine, Proceedings of the Institution of Mechanical Engineers, Part A: Journal of Power and Energy 231 (3) (2017) 197-214. doi:10.1177/0957650917691640,

[40] T. Ghisu, P. Puddu, F. Cambuli, N. Mandas, P. Seshadri, G. T. Parks, Discussion on Performance analysis of Wells turbine blades using the entropy generation minimization method by Shehata, A. S., Saqr, K. M., Xiao, Q., Shahadeh, M. F. and Day, A., Renewable Energy 118 (2018) 386-392. doi:10.1016/j.renene.2017.10.107. 
[41] W. J. McCroskey, The phenomenon of dynamic stall, NASA Technical Memorandum 81264, NASA (March 1981).

[42] F. Menter, Zonal two equations $k-\omega$ turbulence models for aerodynamic flows, in: 24th Fluid Dynamics Conference, Orlando, Florida, USA, 1993.

[43] M. Paderi, P. Puddu, Experimental investigation in a Wells turbine under bi-directional flow, Renewable Energy 57 (2013) 570-576. doi: 10.1016/j.renene.2013.02.016

[44] S. L. Dixon, C. Hall, Fluid Mechanics and Thermodynamics of Turbomachinery, Butterworth Heinemann, Boston, USA, 2010.

[45] T. Ghisu, F. Cambuli, M. Mandas, P. Puddu, P. Seshadri, G. T. Parks, Numerical evaluation of entropy generation in isolated airfoils and Wells turbines, Meccanica, Under review.

[46] M. Torresi, S. Camporeale, G. Pascazio, Detailed CFD analysis of the steady flow in a Wells turbine under incipient and deep stall conditions, Journal of Fluids Engineering, Transactions of the ASME 131 (7) (2009) 0711031-07110317. doi:10.1115/1.3155921.

[47] I. Virdis, T. Ghisu, F. Cambuli, P. Puddu, Dynamic interaction between OWC system and Wells turbine: a comparison between CFD and lumped parameter model, Renewable Energy, Under review.

[48] Y. Kinoue, T. Setoguchi, T. H. Kim, K. Kaneko, M. Inoue, Mechanism of hysteretic characteristics of Wells turbine for wave power conversion, Journal of Fluids Engineering 125 (2) (2003) 302-307. doi:10.1115/1.1538629.

[49] J. D. Denton, W. N. Dawes, Computational fluid dynamics for turbomachinery design, Proceedings of the Institution of Mechanical Engineers, Part C: Journal of Mechanical Engineering Science 213 (2) (1998) 107-124. 
[50] L. M. C. Gato, M. Webster, An experimental investigation into the effect of rotor blade sweep on the performance of the variable-pitch Wells turbine, Proceedings of the Institution of Mechanical Engineers, Part A: Journal of Power and Energy 215.

[51] J. Watterson, S. Raghunathan, Computed effects of tip clearance on the Wells turbine performance, in: Aerospace Sciences Meeting \& Exhibit, Vol. 35th, American Institute of Aeronautics and Astronautics, Inc., Reno, Nevada, USA, 1997.

[52] M. Takao, T. Setoguchi, Y. Kinoue, K. Kaneko, Wells turbine with end plates for wave energy conversion, Ocean Engineering 34 (11) (2007) 1790 -1795 .

[53] Y. Cui, B. Hyun, Numerical study on Wells turbine with penetrating blade tip treatments for wave energy conversion, International Journal of Naval Architecture and Ocean Engineering 8 (5) (2016) 456-465. doi:10.1016/j.ijnaoe.2016.05.009.

[54] P. Halder, A. Samad, D. Tjevenin, Improved design of a Wells turbine for higher operating range, Renewable Energy 106 (2017) 122-134. doi:10.1016/j.renene.2017.01.012 